\title{
TRAWL COD-END MESH SELECTIVITY FOR SOME FISHES OF NORTH-WESTERN AUSTRALIA
}

\author{
HSI-CHIANG LIU ${ }^{1}$, K.J. SAINSBURY ${ }^{2}$ and TAI-SHENG CHIU ${ }^{3}$ \\ ${ }^{1}$ Institute of Oceanography, National Taiwan University, Taipei (Taiwan) \\ ${ }^{2}$ Division of Fisheries Research, CSIRO Marine Laboratories, P.O. Box 21, Cronulla, \\ N.S.W. 2230 (Australia) \\ ${ }^{3}$ Department of Zoology, National Taiwan University, Taipei (Taiwan)
}

(Accepted for publication 26 October 1984)

\section{ABSTRACT}

Liu, Hsi-Chiang, Sainsbury, K.J. and Chiu, Tai-Sheng, 1985. Trawl cod-end mesh selectivity for some fishes of north-western Australia. Fish. Res., 3: 105-129.

A codend mesh selectivity experiment was conducted using the covered cod-end technique on the continental shelf of northern Australia. Cod-end (stretched) mesh sizes of $40,51,55,70$ and $100 \mathrm{~mm}$ were tested with a $30-\mathrm{mm}$-mesh cover bag.

Mesh selection parameters were estimated for 28 species by fitting a logistic curve to the size-specific escapement data, and a gross selection rate was calculated for an additional 27 species. Some species (notably Lutjanus spp., Pristipomoides spp. and Plectorhynchus pictus) were largely retained by all codend mesh sizes tested, while others (notably Loligo spp.) largely escaped all the cod-ends tested. The common assumption that the selection factor does not alter with mesh size was accepted for Nemipterus nematopus, Parupeneus pleurospilus and Lutjanus vittus, but rejected for Saurida undosquamis. Dependence of the selection factor upon mesh size for $S$. undosquamis was due to a non-zero intercept in the relationship between fish size at $50 \%$ escapement and mesh size. The general implications of dependence of selection factor on mesh size are discussed.

\section{INTRODUCTION}

The Taiwanese demersal pair-trawl fishery off Australia began in 1971, when $3471 \mathrm{t}$ of fish was taken from the continental shelf adjacent to Darwin (Liu et al., 1978). In 1972, the fishery had expanded northward to cover most of the Arafura Sea, resulting in a catch of about $74000 \mathrm{t}$. Further expansion of the fishery southward to the Timor Sea and the North West Shelf in the following years provided the maximum catch so far of $83 \mathbf{3 8 1}$ $t$ in 1974. Fishing activities gradually decreased subsequently, with about $50000 \mathrm{t}$ per year being caught between 1977 and 1980 (Fig. 1).

After the declaration of the 200-mile Australian Fishing Zone (AFZ) on 1 November 1979, the Taiwanese fleet continued operation under an 
annual bilateral agreement. From November 1979 to October 1980, 120 trawlers were licensed to take a demersal catch of $27500 \mathrm{t}$.

Regulation of cod-end mesh size is an important aspect of trawl fishery management, and determination of the best mesh size requires, amongst other things, knowledge of the relationship between cod-end mesh size and the probability of retention of fish. To help determine the optimal mesh size for the fishery, scientists from Taiwan and Australia cooperated in a joint research project to determine mesh selectivity for a number of fish species caught in the AFZ. A minimum mesh size of $60 \mathrm{~mm}$ is currently in force (Sainsbury, 1984).

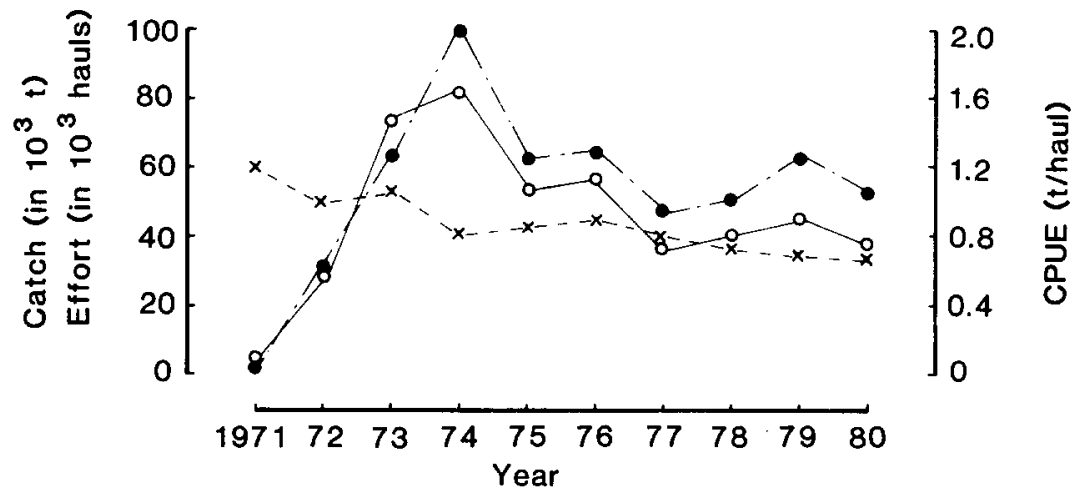

Fig. 1. Estimated annual catch, annual effort, and catch per unit effort (cpue) of Taiwanese trawlers adjacent to northern Australia, both inside and beyond the 200-mile Australian Fishing Zone. $\circ=$ catch in $10^{3} \mathrm{t} ; \bullet=$ effort in $10^{3} 3 \cdot \mathrm{h}$ hauls; $\mathrm{x}=$ cpue in tonnes per 3-h hauls.

This study was conducted during two cruises of the Taiwan Fisheries Research Institute vessel "R.V. Hai-Kong" in north-western Australia during 1981.

The retention parameters of six different cod-end mesh sizes were determined using the cod-end cover method (Pope, 1975). These parameters were used to examine the commonly held assumption of direct proportionality between mean size of retained fish and mesh size (e.g. Jensen, 1949; Beverton and Holt, 1957, pp. 221-232), which results in independence of the selection factor (Pope, 1975) and mesh size. Clark (1963) also tested this assumption, and showed that the selection factor increased with mesh size for silver hake.

\section{METHODS}

Research vessel

"R.V. Hai-Kong" is a 56.6-m stern trawler with a 2200-B.H.P. main engine and a maximum cruising speed of 13.5 knots. All trawls were conducted at 3 knots. 


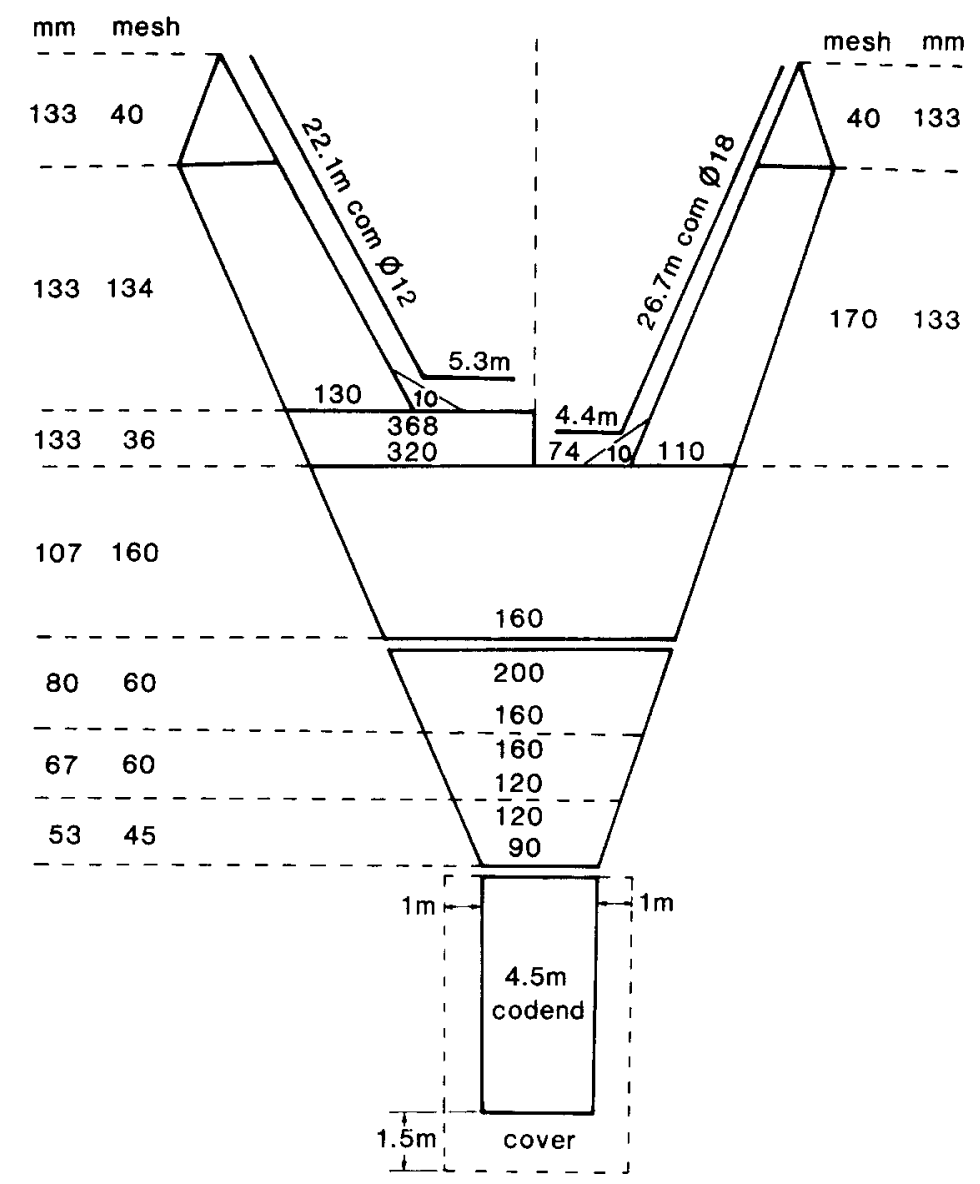

Fig. 2. Trawl net and cod-end cover bag construction.

Experimental nets

A plan of the experimental net is shown in Fig. 2. The cod-end was enclosed by a $30-\mathrm{mm}$ mesh cover net $2 \mathrm{~m}$ wider in radius and $1.5 \mathrm{~m}$ longer than the codend.

The mesh opening of each experimental cod-end was assessed by averaging the longitudinal inner diameter of 30 cod-end meshes while a $5-\mathrm{kg}$ weight was hung from the mesh being measured (Aoyoma, 1961). The six cod-ends used in the experiment had mesh openings of $40,51,55,70,83$ and 100 $\mathrm{mm}$ (Table I). The netting yarn of each is shown in Table I.

Time and area of operation

The experiments were conducted between 9 March and 13 April 1981 in areas of high fishing activity (Anon., 1979, 1980). The start locations of the chosen trawl sites are shown in Fig. 3. 


\section{TABLE I}

The inner diameter and material of cover net and cod-end

\begin{tabular}{llcll}
\hline Cod-end & Sample size & $\begin{array}{l}\text { Inner diameter } \\
(\mathrm{mm})\end{array}$ & $\begin{array}{l}\text { Standard deviation } \\
(\mathrm{mm})\end{array}$ & $\begin{array}{l}\text { Net material } \\
(\text { Rtex })\end{array}$ \\
\hline A & 30 & 39.79 & 2.07 & 1025 \\
B & 30 & 50.74 & 1.54 & 1265 \\
C & 30 & 54.68 & 1.86 & 1900 \\
D & 30 & 70.41 & 2.80 & 1900 \\
E & 30 & 82.92 & 3.27 & 2530 \\
F & 30 & 100.23 & 6.79 & 2530 \\
& & 30.30 & 0.96 & 1025 \\
Cover & 30 & & &
\end{tabular}

Two to six trawls were completed during daylight hours of each day, and generally two consecutive hauls were made with each cod-end mesh size. Trawl duration ranged from 31 to $57 \mathrm{~min}$, with $65 \%$ of trawls in the range 40-45 min. The number of hauls using each cod-end mesh size is shown in Table II.

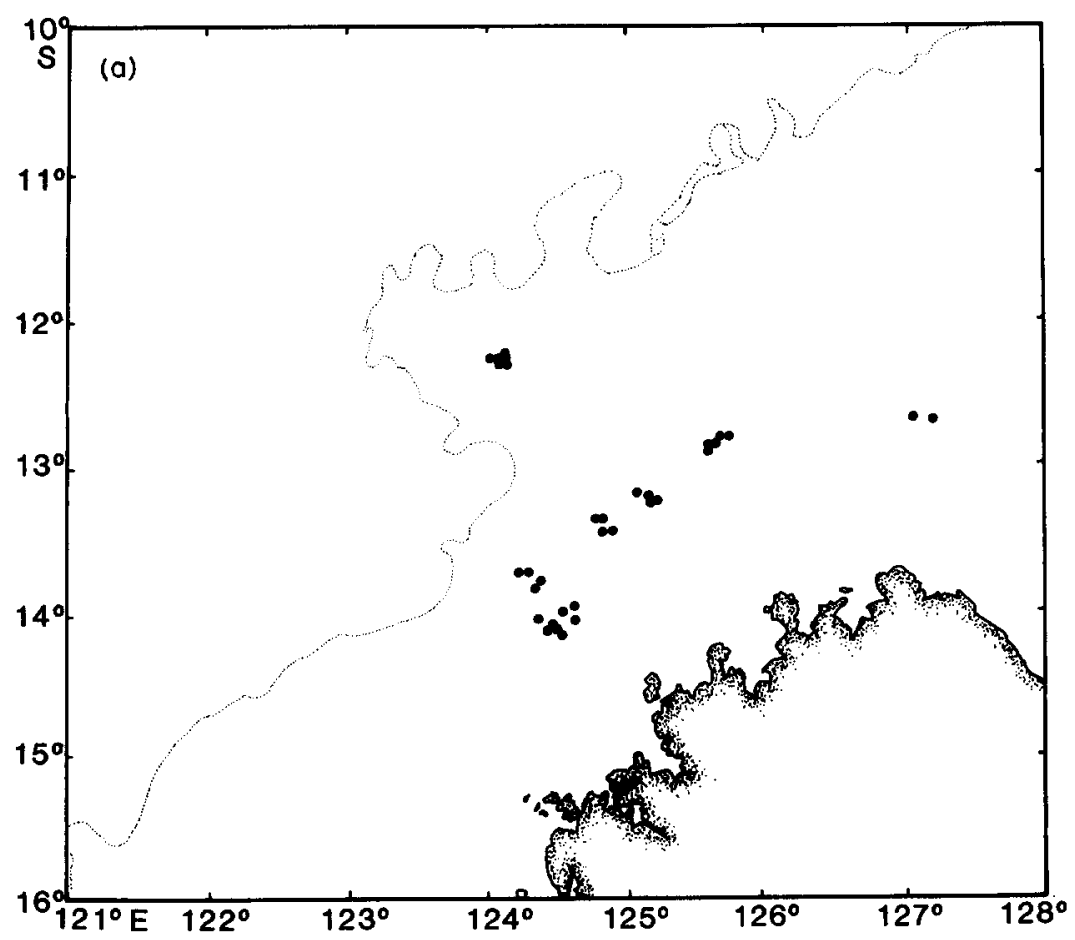

Fig. 3. Sampling locations for mesh selectivity experiments.

(a) The Kimberly Region. (b) The North West Shelf. 


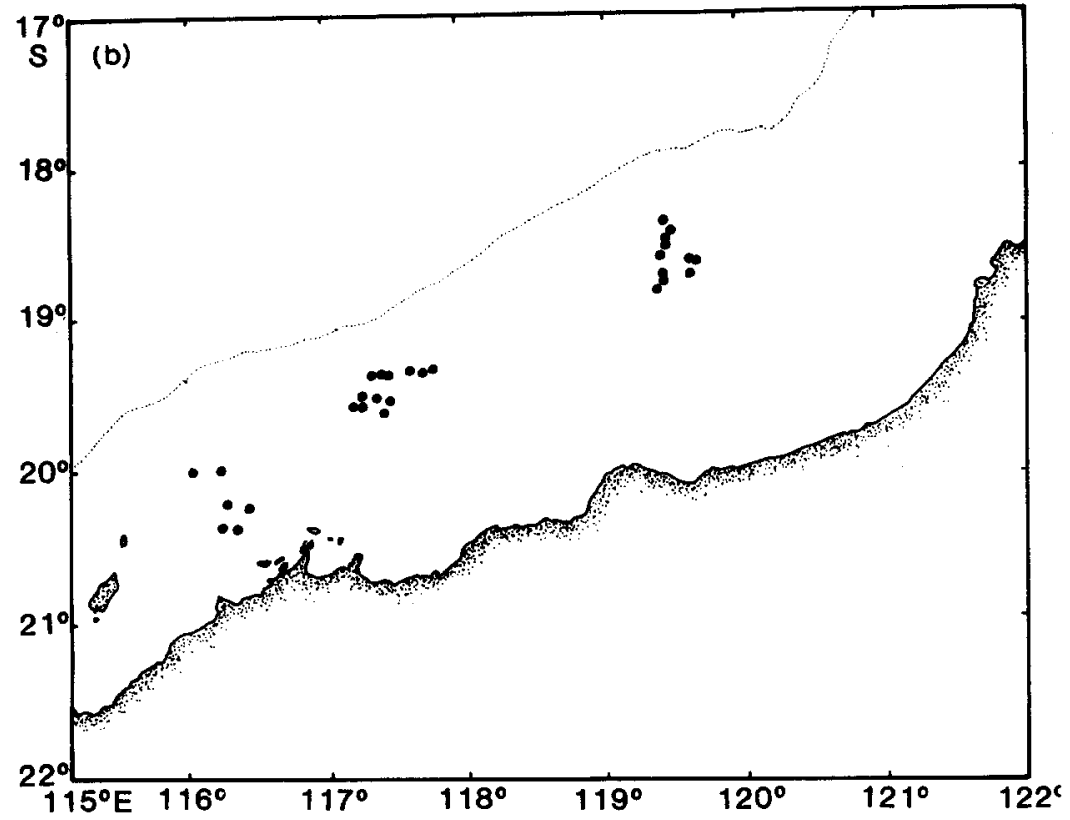

TABLE II

The number of hauls made with each cod-end mesh size in the Kimberly region (Fig. 3a) and the North West Shelf (Fig. 3b)

\begin{tabular}{lrrrrrl}
\hline \multicolumn{7}{c}{ Cod-end mesh size $(\mathrm{mm})$} \\
\cline { 2 - 7 } & 40 & 51 & 55 & 70 & 83 & 100 \\
\hline Kimberly region & 8 & 6 & 5 & 15 & - & 4 \\
N.W. Shelf & 7 & 5 & 9 & - & 6 & 2 \\
Total & 15 & 11 & 14 & 15 & 6 & 6 \\
\hline
\end{tabular}

Measurements of catch

Length-frequency measurements were made for the major species in the cod-end and cover net after each trawl. Fork length was measured for most species, but in some cases (Table III) total length, snout to anus length or mantle length was used. Usually the entire catch of a species was measured, but some large catches of leiognathids and small mullids were subsampled.

Analysis

The proportion of fish in each 1-cm size class retained by a cod-end (retention) was calculated for each species and codend mesh size. 


\section{TABLE III}

The parameters of the mesh selection curves for each cod-end mesh size and the dimension measured on each species ( $T L=$ total length, $F L=$ fork length, $M L=$ mantle length). The selectivity parameters $(L, S S, S R$ and $S)$ are defined in the analysis section of the text

\begin{tabular}{|c|c|c|c|c|c|c|c|c|}
\hline Species & $\begin{array}{l}\text { Dimen- } \\
\text { sion }\end{array}$ & $\begin{array}{l}\text { Mesh } \\
\text { size } \\
(\mathrm{mm})\end{array}$ & $\begin{array}{l}L_{0.25} \\
(\mathrm{~mm})\end{array}$ & $\begin{array}{l}L_{0.30} \\
(\mathrm{~mm})\end{array}$ & $\begin{array}{l}L_{0,75} \\
(\mathrm{~mm})\end{array}$ & $\begin{array}{l}S S \\
(\mathrm{~mm})\end{array}$ & $S R$ & $S$ \\
\hline Nemipterus nematopus & $F L$ & $\begin{array}{r}40 \\
51 \\
54 \\
70 \\
83 \\
100\end{array}$ & $\begin{array}{r}73.5 \\
94.7 \\
43.2 \\
176.3 \\
131.5\end{array}$ & $\begin{array}{r}85.5 \\
115.3 \\
118.3 \\
187.5 \\
217.7\end{array}$ & $\begin{array}{r}89.9 \\
135.8 \\
203.4 \\
198.7 \\
286.7\end{array}$ & $\begin{array}{r}16.4 \\
41.1 \\
160.2 \\
22.4 \\
155.2\end{array}$ & $\begin{array}{l}0.12 \\
0.30 \\
0.38 \\
0.94 \\
0.77 \\
0.99\end{array}$ & $\begin{array}{l}2.1 \\
2.3 \\
2.2 \\
2.7 \\
2.6\end{array}$ \\
\hline N. tolu & $F L$ & $\begin{array}{l}51 \\
55 \\
70\end{array}$ & 123.7 & 135.6 & 147.5 & 23.8 & $\begin{array}{l}0.03 \\
0.10 \\
0.99\end{array}$ & 2.5 \\
\hline$N$. hexadon & $F L$ & $\begin{array}{r}51 \\
70 \\
100\end{array}$ & 171.3 & 175.6 & 187.4 & 16.1 & $\begin{array}{l}0.01 \\
0.64 \\
0.99\end{array}$ & 2.5 \\
\hline N. peronii & & $\begin{array}{r}40 \\
55 \\
70 \\
100\end{array}$ & $\begin{array}{l}139.0 \\
136.9\end{array}$ & $\begin{array}{l}153.5 \\
150.8\end{array}$ & $\begin{array}{l}168.1 \\
164.7\end{array}$ & $\begin{array}{l}29.1 \\
27.8\end{array}$ & $\begin{array}{l}0.05 \\
0.69 \\
0.24 \\
0.84\end{array}$ & $\begin{array}{l}2.8 \\
2.1\end{array}$ \\
\hline N. virgatus & $F L$ & $\begin{array}{r}51 \\
55 \\
70 \\
100\end{array}$ & $\begin{array}{r}69.0 \\
81.8 \\
138.1\end{array}$ & $\begin{array}{l}105.1 \\
109.2 \\
163.5\end{array}$ & $\begin{array}{l}141.2 \\
137.6 \\
188.8\end{array}$ & $\begin{array}{l}72.2 \\
55.8 \\
50.7\end{array}$ & $\begin{array}{l}0.25 \\
0.15 \\
0.57 \\
0.46\end{array}$ & $\begin{array}{l}2.1 \\
2.0 \\
2.3\end{array}$ \\
\hline N. tambuloides & $F L$ & $\begin{array}{r}40 \\
51 \\
55 \\
70 \\
100\end{array}$ & $\begin{array}{c}102.6 \\
118.9 \\
158.1 \\
\text {, }\end{array}$ & $\begin{array}{l}121.7 \\
142.3 \\
169.1\end{array}$ & $\begin{array}{c}140.8 \\
165.7 \\
180.1\end{array}$ & $\begin{array}{l}38.2 \\
46.8 \\
\\
22.0\end{array}$ & $\begin{array}{l}0.39 \\
0.51 \\
0.55 \\
0.84 \\
0.94\end{array}$ & $\begin{array}{l}3.1 \\
2.8 \\
\\
2.4\end{array}$ \\
\hline N. bathybus & $F L$ & $\begin{array}{r}40 \\
55 \\
70 \\
100\end{array}$ & $\begin{array}{l}69.3 \\
83.7\end{array}$ & $\begin{array}{r}77.3 \\
119.7\end{array}$ & $\begin{array}{r}85.4 \\
155.8\end{array}$ & $\begin{array}{l}16.1 \\
72.1\end{array}$ & $\begin{array}{l}0.15 \\
0.27 \\
0.95 \\
0.53\end{array}$ & $\begin{array}{l}1.9 \\
2.2\end{array}$ \\
\hline Saurida undosquamis & $F L$ & $\begin{array}{r}40 \\
51 \\
55 \\
70 \\
83 \\
100\end{array}$ & $\begin{array}{l}166.3 \\
138.7 \\
156.0 \\
173.2\end{array}$ & $\begin{array}{l}180.1 \\
196.7 \\
210.1 \\
257.7\end{array}$ & $\begin{array}{l}193.9 \\
262.5 \\
264.2 \\
342.2\end{array}$ & $\begin{array}{r}27.6 \\
123.8 \\
108.2 \\
168.9\end{array}$ & $\begin{array}{l}0.35 \\
0.57 \\
0.27 \\
0.76 \\
0.79 \\
0.54\end{array}$ & $\begin{array}{l}4.5 \\
3.9 \\
3.8 \\
3.7\end{array}$ \\
\hline
\end{tabular}


TABLE III (continued)

\begin{tabular}{|c|c|c|c|c|c|c|c|c|}
\hline Species & $\begin{array}{l}\text { Dimen- } \\
\text { sion }\end{array}$ & $\begin{array}{l}\text { Mesh } \\
\text { size } \\
(\mathrm{mm})\end{array}$ & $\begin{array}{l}L_{0.25} \\
(\mathrm{~mm})\end{array}$ & $\begin{array}{l}L_{0 . s 0} \\
(\mathrm{~mm})\end{array}$ & $\begin{array}{l}L_{0.75} \\
(\mathrm{~mm})\end{array}$ & $\begin{array}{l}S S \\
(\mathrm{~mm})\end{array}$ & $S R$ & $S$ \\
\hline S. filamentosa & $F L$ & $\begin{array}{r}51 \\
55 \\
70 \\
100\end{array}$ & $\begin{array}{l}97.1 \\
66.1\end{array}$ & $\begin{array}{l}155.2 \\
157.1\end{array}$ & $\begin{array}{l}213.3 \\
248.3\end{array}$ & $\begin{array}{l}116.2 \\
182.2\end{array}$ & $\begin{array}{l}0.03 \\
0.10 \\
0.26 \\
0.93\end{array}$ & $\begin{array}{l}2.8 \\
2.2\end{array}$ \\
\hline S. micropectoralis & $F L$ & $\begin{array}{r}55 \\
70 \\
100\end{array}$ & $\begin{array}{l}113.8 \\
215.3 \\
253.5\end{array}$ & $\begin{array}{l}152.9 \\
232.1 \\
274.4\end{array}$ & $\begin{array}{l}167.3 \\
248.9 \\
295.2\end{array}$ & $\begin{array}{l}53.3 \\
33.6 \\
41.7\end{array}$ & $\begin{array}{l}0.39 \\
0.23 \\
0.64\end{array}$ & $\begin{array}{l}2.8 \\
3.3 \\
2.7\end{array}$ \\
\hline Trachinocephalus myops & $F L$ & $\begin{array}{l}40 \\
55 \\
70\end{array}$ & $\begin{array}{l}98.4 \\
95.6\end{array}$ & $\begin{array}{l}104.4 \\
107.7\end{array}$ & $\begin{array}{l}106.5 \\
119.7\end{array}$ & $\begin{array}{l}08.1 \\
24.1\end{array}$ & $\begin{array}{l}0.24 \\
0.57 \\
0.88\end{array}$ & $\begin{array}{l}2.6 \\
2.0\end{array}$ \\
\hline Lutjanus malabarius & $T L$ & $\begin{array}{r}70 \\
83 \\
100\end{array}$ & & & & & $\begin{array}{l}0.02 \\
0.07 \\
0.01\end{array}$ & \\
\hline S. sebae & $T L$ & $\begin{array}{r}83 \\
100\end{array}$ & & & & & $\begin{array}{l}0.02 \\
0.08\end{array}$ & \\
\hline L. russelli & $T L$ & $\begin{array}{r}70 \\
100\end{array}$ & & & & & $\begin{array}{l}0.02 \\
0.45\end{array}$ & \\
\hline L. argentimaculatus & $T L$ & 100 & & & & & 0.01 & \\
\hline Therapon theraps & $T L$ & $\begin{array}{r}55 \\
70 \\
100\end{array}$ & $\begin{array}{r}116.8 \\
75.9\end{array}$ & $\begin{array}{l}125.5 \\
113.8\end{array}$ & $\begin{array}{l}134.2 \\
151.7\end{array}$ & $\begin{array}{l}17.4 \\
75.8\end{array}$ & $\begin{array}{l}0.36 \\
0.36 \\
0.93\end{array}$ & $\begin{array}{l}2.5 \\
1.6\end{array}$ \\
\hline Priacanthus hamrur & $T L$ & $\begin{array}{r}40 \\
70 \\
83 \\
100\end{array}$ & 178.1 & 191.6 & 205.0 & 26.9 & $\begin{array}{l}0.04 \\
0.18 \\
0.45 \\
0.33\end{array}$ & 1.9 \\
\hline$P$. tayenus & $T L$ & $\begin{array}{l}70 \\
83\end{array}$ & & & & & $\begin{array}{l}0.02 \\
0.09\end{array}$ & \\
\hline Pentaprion longimanus & $F L$ & $\begin{array}{r}40 \\
50 \\
55 \\
70 \\
83 \\
100\end{array}$ & $\begin{array}{l}45.0 \\
52.9 \\
99.4\end{array}$ & $\begin{array}{r}54.1 \\
78.9 \\
114.6\end{array}$ & $\begin{array}{r}73.4 \\
104.9 \\
120.2\end{array}$ & $\begin{array}{l}28.4 \\
52.0 \\
20.8\end{array}$ & $\begin{array}{l}0.05 \\
0.24 \\
0.59 \\
0.98 \\
0.94 \\
0.97\end{array}$ & $\begin{array}{l}1.4 \\
1.6 \\
2.1\end{array}$ \\
\hline Lutjanus vittus & $T L$ & $\begin{array}{r}51 \\
55 \\
70 \\
83 \\
100\end{array}$ & $\begin{array}{l}158.3 \\
121.2 \\
178.8 \\
247.2 \\
239.8\end{array}$ & $\begin{array}{l}160.4 \\
130.6 \\
194.0 \\
258.3 \\
251.5\end{array}$ & $\begin{array}{l}162.5 \\
140.0 \\
209.1 \\
269.4 \\
263.3\end{array}$ & $\begin{array}{r}4.2 \\
18.8 \\
30.3 \\
22.2 \\
23.5\end{array}$ & $\begin{array}{l}0.01 \\
0.05 \\
0.19 \\
0.19 \\
0.70\end{array}$ & $\begin{array}{l}3.2 \\
2.4 \\
2.8 \\
3.1 \\
2.5\end{array}$ \\
\hline
\end{tabular}


TABLE III (continued)

\begin{tabular}{|c|c|c|c|c|c|c|c|c|}
\hline Species & $\begin{array}{l}\text { Dimen- } \\
\text { sion }\end{array}$ & $\begin{array}{l}\text { Mesh } \\
\text { size } \\
(\mathrm{mm})\end{array}$ & $\begin{array}{l}L_{0.25} \\
(\mathrm{~mm})\end{array}$ & $\begin{array}{l}L_{0.80} \\
(\mathrm{~mm})\end{array}$ & $\begin{array}{l}L_{0.75} \\
(\mathrm{~mm})\end{array}$ & $\begin{array}{l}S S \\
(\mathrm{~mm})\end{array}$ & $S R$ & $S$ \\
\hline Pomadasys hasta & $F L$ & $\begin{array}{r}70 \\
100\end{array}$ & 222.9 & 251.2 & 271.0 & 48.1 & $\begin{array}{l}0.03 \\
0.19\end{array}$ & 2.5 \\
\hline Epinephelus spp. & $T L$ & $\begin{array}{r}55 \\
70 \\
83 \\
100\end{array}$ & 147.0 & 164.9 & 182.8 & 35.8 & $\begin{array}{l}0.04 \\
0.38 \\
0.07 \\
0.37\end{array}$ & 2.3 \\
\hline Lepidotrigla spiloptera & $F L$ & $\begin{array}{r}55 \\
70 \\
100\end{array}$ & $\begin{array}{l}120.9 \\
152.3\end{array}$ & $\begin{array}{l}139.1 \\
171.0\end{array}$ & $\begin{array}{l}157.2 \\
189.8\end{array}$ & $\begin{array}{l}36.3 \\
37.5\end{array}$ & $\begin{array}{l}0.05 \\
0.09 \\
0.29\end{array}$ & $\begin{array}{l}2.0 \\
1.7\end{array}$ \\
\hline Arius thalassinus & $F L$ & $\begin{array}{r}70 \\
100\end{array}$ & & & & & $\begin{array}{l}0.02 \\
0.06\end{array}$ & \\
\hline Lethrinus spp. & $F L$ & $\begin{array}{r}70 \\
83 \\
100\end{array}$ & & & & & $\begin{array}{l}0.01 \\
0.05 \\
0.13\end{array}$ & \\
\hline Pristipomoides spp. & $F L$ & $\begin{array}{r}51 \\
70 \\
100\end{array}$ & & & & & $\begin{array}{l}0.01 \\
0.03 \\
0.39\end{array}$ & \\
\hline Plectorhynchus pictus & $F L$ & 100 & & & & & 0.01 & \\
\hline Platax batavianus & $F L$ & 100 & & & & & 0.01 & \\
\hline Carangoides spp. & $F L$ & $\begin{array}{r}55 \\
70 \\
83 \\
100\end{array}$ & $\begin{array}{r}61.8 \\
\\
168.4 \\
118.3\end{array}$ & $\begin{array}{r}77.3 \\
\\
176.9 \\
137.1\end{array}$ & $\begin{array}{r}92.9 \\
185.5 \\
156.0\end{array}$ & $\begin{array}{l}31.1 \\
\\
17.1 \\
37.7\end{array}$ & $\begin{array}{l}0.03 \\
0.09 \\
0.05 \\
0.44\end{array}$ & $\begin{array}{l}1.3 \\
\\
2.1 \\
1.4\end{array}$ \\
\hline Decapterus maruadsi & $F L$ & $\begin{array}{r}55 \\
70 \\
83 \\
100\end{array}$ & & & & & $\begin{array}{l}0.99 \\
0.73 \\
0.96 \\
0.69\end{array}$ & \\
\hline Gathanodon speciosus & $F L$ & $\begin{array}{r}70 \\
100\end{array}$ & & & & & $\begin{array}{l}0.04 \\
0.35\end{array}$ & \\
\hline Parupeneus pleurospilus & $F L$ & $\begin{array}{r}40 \\
51 \\
55 \\
70 \\
83 \\
100\end{array}$ & $\begin{array}{r}53.1 \\
125.7 \\
88.1 \\
159.8 \\
191.4 \\
191.9\end{array}$ & $\begin{array}{r}86.6 \\
135.9 \\
117.2 \\
181.7 \\
213.5 \\
217.0\end{array}$ & $\begin{array}{l}120.1 \\
139.6 \\
146.2 \\
202.6 \\
235.6 \\
242.0\end{array}$ & $\begin{array}{l}67.0 \\
13.9 \\
58.1 \\
43.8 \\
44.2 \\
50.1\end{array}$ & $\begin{array}{l}0.52 \\
0.22 \\
0.69 \\
0.48 \\
0.41 \\
0.87\end{array}$ & $\begin{array}{l}2.2 \\
2.7 \\
2.0 \\
2.6 \\
2.6 \\
2.2\end{array}$ \\
\hline
\end{tabular}


TABLE III (continued)

\begin{tabular}{|c|c|c|c|c|c|c|c|c|}
\hline Species & $\begin{array}{l}\text { Dimen- } \\
\text { sion }\end{array}$ & $\begin{array}{l}\text { Mesh } \\
\text { size } \\
(\mathrm{mm})\end{array}$ & $\begin{array}{l}L_{0.25} \\
(\mathrm{~mm})\end{array}$ & $\begin{array}{l}L_{0.50} \\
(\mathbf{m m})\end{array}$ & $\begin{array}{l}L_{0.75} \\
(\mathrm{~mm})\end{array}$ & $\begin{array}{l}S S \\
(\mathrm{~mm})\end{array}$ & $S R$ & $S$ \\
\hline Upeneus spp. & $F L$ & $\begin{array}{r}40 \\
51 \\
55 \\
70 \\
83 \\
100\end{array}$ & $\begin{array}{r}98.5 \\
105.7 \\
105.2 \\
115.4\end{array}$ & $\begin{array}{l}102.0 \\
123.9 \\
124.0 \\
126.9\end{array}$ & $\begin{array}{l}105.5 \\
142.1 \\
142.9 \\
138.4\end{array}$ & $\begin{array}{l}07.0 \\
36.4 \\
37.7 \\
23.0\end{array}$ & $\begin{array}{l}0.10 \\
0.79 \\
0.57 \\
0.09 \\
0.96 \\
1.00\end{array}$ & $\begin{array}{l}2.6 \\
2.4 \\
2.3 \\
1.8\end{array}$ \\
\hline Formio niger & $F L$ & 100 & & & & & 0.00 & \\
\hline Psenopsis anomala & $F L$ & $\begin{array}{r}55 \\
70 \\
100\end{array}$ & 60.9 & 78.8 & 96.6 & 35.7 & $\begin{array}{l}0.14 \\
0.04 \\
0.26\end{array}$ & 1.4 \\
\hline Signanus canaliculatus & $F L$ & 100 & & & & & 0.28 & \\
\hline Hapologenys kishinouyei & $F L$ & 55 & & & & & 0.08 & \\
\hline Acropoma japonicum & $F L$ & $\begin{array}{l}40 \\
51 \\
55 \\
83\end{array}$ & $\begin{array}{l}58.6 \\
95.2\end{array}$ & $\begin{array}{r}65.6 \\
115.8\end{array}$ & $\begin{array}{r}72.6 \\
136.3\end{array}$ & $\begin{array}{l}14.0 \\
41.1\end{array}$ & $\begin{array}{l}0.32 \\
1.00 \\
0.94 \\
0.84\end{array}$ & $\begin{array}{l}1.6 \\
2.3\end{array}$ \\
\hline Myripristus murdjan & $F L$ & 100 & & & & & 0.00 & \\
\hline Leioganthus fasciatus & $F L$ & 70 & & & & & 0.41 & \\
\hline Spyraena forsteri & $F L$ & $\begin{array}{r}51 \\
55 \\
70 \\
83 \\
100\end{array}$ & 151.6 & 168.2 & 184.7 & 33.1 & $\begin{array}{l}0.20 \\
0.54 \\
0.88 \\
0.75 \\
0.80\end{array}$ & 3.0 \\
\hline Leioganthus spp. & $F L$ & $\begin{array}{r}40 \\
51 \\
55 \\
70 \\
100\end{array}$ & $\begin{array}{l}51.7 \\
\\
40.2 \\
44.8\end{array}$ & $\begin{array}{l}66.4 \\
\\
52.3 \\
51.1\end{array}$ & $\begin{array}{l}81.0 \\
\\
64.3 \\
57.4\end{array}$ & $\begin{array}{l}29.3 \\
\\
24.1 \\
12.6\end{array}$ & $\begin{array}{l}0.64 \\
0.87 \\
0.51 \\
0.55 \\
0.94\end{array}$ & $\begin{array}{l}1.7 \\
1.0 \\
0.7\end{array}$ \\
\hline Johnieops vogleri & $T L$ & $\begin{array}{l}40 \\
55 \\
70\end{array}$ & $\begin{array}{l}107.0 \\
155.4\end{array}$ & $\begin{array}{l}111.4 \\
167.7\end{array}$ & $\begin{array}{l}115.8 \\
179.9\end{array}$ & $\begin{array}{r}8.8 \\
24.5\end{array}$ & $\begin{array}{l}0.12 \\
0.64 \\
0.18\end{array}$ & $\begin{array}{l}2.8 \\
3.1\end{array}$ \\
\hline Harpodon translucens & $T L$ & $\begin{array}{l}40 \\
51 \\
55 \\
83\end{array}$ & $\begin{array}{l}57.9 \\
88.3\end{array}$ & $\begin{array}{l}85.4 \\
92.7\end{array}$ & $\begin{array}{r}112.9 \\
97.1\end{array}$ & $\begin{array}{r}55.0 \\
8.8\end{array}$ & $\begin{array}{l}0.20 \\
0.50 \\
0.90 \\
0.86\end{array}$ & $\begin{array}{l}2.1 \\
1.7\end{array}$ \\
\hline
\end{tabular}


TABLE III (continued)

\begin{tabular}{|c|c|c|c|c|c|c|c|c|}
\hline Species & $\begin{array}{l}\text { Dimen- } \\
\text { sion }\end{array}$ & $\begin{array}{l}\text { Mesh } \\
\text { size } \\
(\mathrm{mm})\end{array}$ & $\begin{array}{l}L_{0.25} \\
(\mathrm{~mm})\end{array}$ & $\begin{array}{l}L_{0.30} \\
(\mathrm{~mm})\end{array}$ & $\begin{array}{l}L_{0,75} \\
(\mathrm{~mm})\end{array}$ & $\begin{array}{l}S S \\
(\mathrm{~mm})\end{array}$ & $S R$ & $S$ \\
\hline \multirow[t]{6}{*}{ Loligo spp. } & \multirow[t]{6}{*}{$M L$} & 40 & 9.0 & 28.5 & 58.1 & 49.1 & 0.43 & 0.7 \\
\hline & & 51 & 33.9 & 42.5 & 51.1 & 17.2 & 0.78 & 0.8 \\
\hline & & 55 & & & & & 0.91 & \\
\hline & & 70 & & & & & 0.72 & \\
\hline & & 83 & & & & & 0.89 & \\
\hline & & 100 & & & & & 0.96 & \\
\hline \multirow[t]{2}{*}{ Sepia spp. } & \multirow[t]{2}{*}{$M L$} & 40 & & & & & 0.20 & \\
\hline & & 55 & & & & & 0.50 & \\
\hline \multirow[t]{4}{*}{ Cyanoglossus spp. } & \multirow[t]{4}{*}{$T L$} & 55 & & & & & 0.11 & \\
\hline & & 70 & & & & & 0.16 & \\
\hline & & 83 & & & & & 0.10 & \\
\hline & & 100 & & & & & 0.15 & \\
\hline \multirow[t]{2}{*}{ Seriolina nigrofasciata } & \multirow[t]{2}{*}{$F L$} & 55 & & & & & 0.67 & \\
\hline & & 100 & & & & & 0.10 & \\
\hline \multirow[t]{3}{*}{ Apogon fasciata } & \multirow[t]{3}{*}{$T L$} & 40 & & & & & 0.09 & \\
\hline & & 83 & & & & & 1.00 & \\
\hline & & 100 & & & & & 1.00 & \\
\hline \multirow[t]{2}{*}{ Adioryx ruber } & \multirow[t]{2}{*}{$F L$} & 83 & & & & & 0.15 & \\
\hline & & 100 & & & & & 0.88 & \\
\hline \multirow[t]{2}{*}{ Argyrops spinifer } & \multirow[t]{2}{*}{$F L$} & 83 & & & & & 0.33 & \\
\hline & & 100 & & & & & 1.00 & \\
\hline \multirow[t]{4}{*}{ Caesio chrysozona } & \multirow[t]{4}{*}{$F L$} & 55 & & & & & 0.16 & \\
\hline & & 70 & & & & & 0.90 & \\
\hline & & 83 & & & & & 0.92 & \\
\hline & & 100 & & & & & 1.00 & \\
\hline \multirow[t]{4}{*}{ Pristipstis jerdoni } & \multirow[t]{4}{*}{$F L$} & 40 & & & & & 0.74 & \\
\hline & & 51 & & & & & 1.00 & \\
\hline & & 55 & & & & & 1.00 & \\
\hline & & 100 & & & & & 1.00 & \\
\hline \multirow[t]{2}{*}{ Choerodon monostigma } & \multirow[t]{2}{*}{$F L$} & 51 & & & & & 0.18 & \\
\hline & & 70 & & & & & 0.60 & \\
\hline
\end{tabular}

The retention proportion was logit transformed (Bliss, 1970, p. 167) and the parameters of a logistic selection curve obtained by weighted linear least-squares regression. Each transformed retention proportion was weighted by the total number of fish measured in determining that proportion. The estimated parameters of the logistic selection curve were used to predict 
fish lengths at $25 \%$ retention $\left(L_{0.25}\right), 50 \%$ retention $\left(L_{0.5}\right)$ and $75 \%$ retention $\left(L_{0.75}\right)$. Following Aoyoma (1961) and Pope (1975), the selection span (SS) is given by $L_{0.75}-L_{0.25}$, and the selection factor $(S)$ is $L_{0.5}$ divided by cod-end mesh size.

For some species the relationship between fish size and retention was poorly defined or inadequately described by a logistic curve for most mesh sizes tested. In these cases, an overall selection rate $(S R)$ foreach mesh size was calculated as the ratio of the number of fish escaping into the covernet to the total number of fish in both cover-net and cod-end (Aoyoma, 1961). The selection-rate lacks generality due to its strong dependence upon the particular distribution of fish sizes encountered during sampling, but is of value in qualitatively indicating species which were mostly retained or which mostly escaped the cod-end mesh sizes employed during the experiment.

Where sufficient data were available, the dependence of the selection factor $(S)$ upon mesh size was examined with Spearman's rank correlation coefficient, and the relationship between $L_{0.5}$ and mesh size was determined by linear least squares regression analysis. It is commonly assumed (e.g. Beverton and Holt, 1957) that the selection factor is independent of mesh size as a result of direct proportionality between $L_{0.5}$ and mesh size.

\section{RESULTS}

Sufficient data were collected to allow the analysis of selection for 54 species (Table III), and the length frequency distributions obtained from each experimental codend and cover bag are shown in Figs. 4-9.

Selection rate was estimated for the 54 species (Table III, Fig. 10), and logistic selection curves could be fitted to the retention data collected from 28 species (Table III). Examples of the fitted logistic selection curves are given for Nemipterus nematopus (Fig. 11), N. virgatus (Fig. 12), Saurida undosquamis (Fig. 13) and Parupeneus pleurospilus (Fig. 14). The fit to the logistic selection curve was generally reasonable, and most cases of a poor fit were due to small sample sizes in the cod-end, cover net or both. The mesh selectivity parameters derived from the fitted logistic curves are given in Table III.

Some species were mostly retained by the trawl, even for the larger codend mesh sizes employed during the experiment (Fig. 10). Lutjanus argentimaculatus, Plectorhynchus pictus, Platax batavinus, Formio niger and Myripristus murdjan were totally retained by all the mesh sizes tested, while Lutjanus sebae was totally retained by mesh sizes up to $70 \mathrm{~mm}$. Pristipomoides spp. were almost all retained by mesh sizes up to and including $83 \mathrm{~mm}$. A large group of species, including Lutjanus malabaricus, L. russelli, Priacanthus tayenus, Pomadasys hasta, Lethrinus spp. (mostly L. lentjan and L. choerorynchus), Arius thalassimus, Gnathonodon speciosus and Argyrops spinifer were totally retained by a codend mesh size of 55 

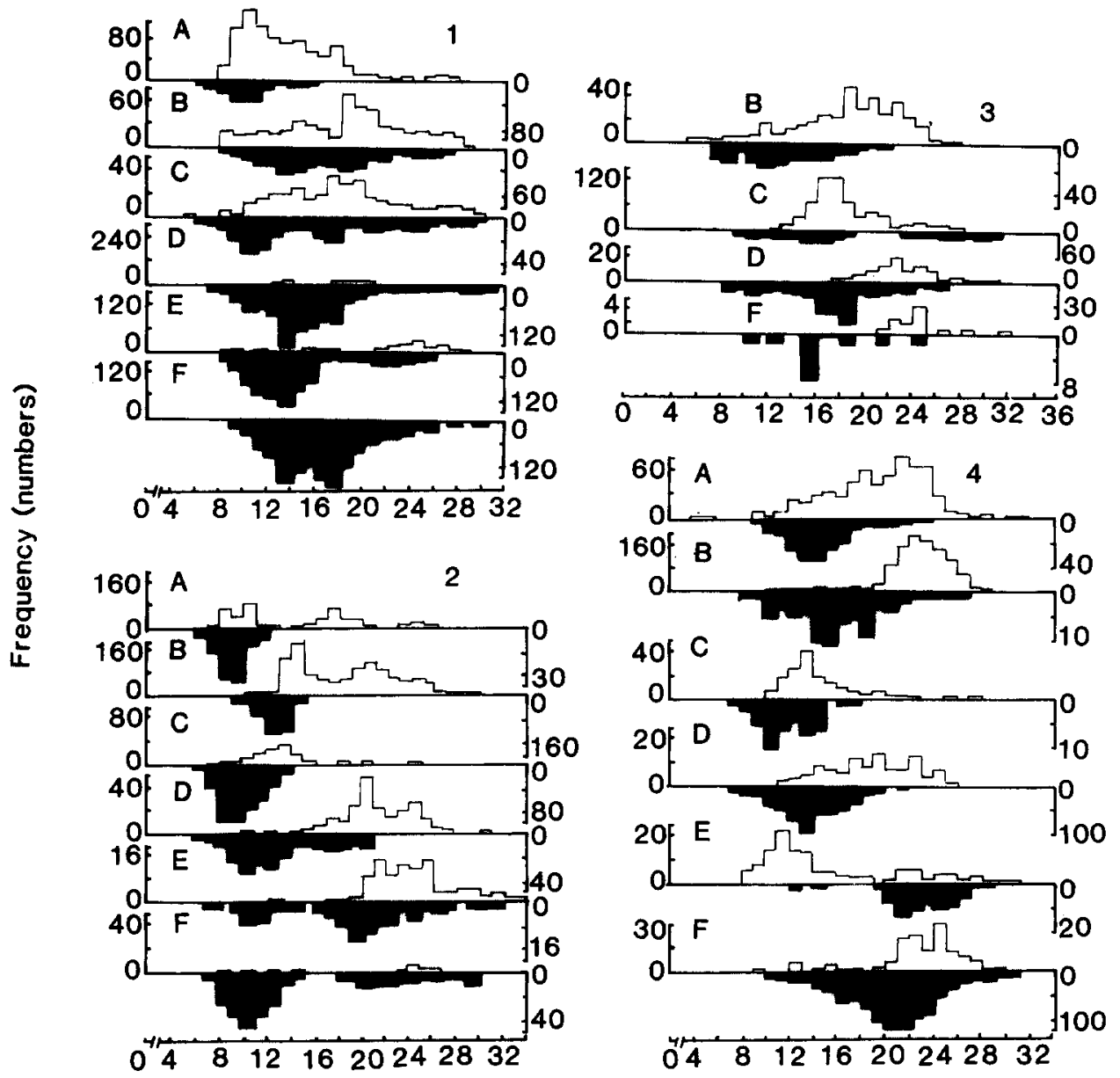

\section{Length (cm)}

Fig. 4. The length frequency distribution of fishes caught by different cod-ends and cover nets. Cod-ends $\mathrm{A}-\mathrm{F}$ are defined in Table I. (1, Nemipterus nematopus; 2 , Nemipterus tambuloides; 3, Nemipterus virgatus; 4, Parupeneus pleurospilus). Abscissa, body length; ordinate, number of fishes. Upper histogram for cod-end catches; lower histogram for cover net catches.

mm. Saurida micropectoralis, Epinephalus spp. and Carangoides spp. were totally retained by a $51-\mathrm{mm}$ cod-end mesh.

A smaller group of species mostly escaped through the meshes of most cod-ends tested (Fig. 10). Most individuals of Loligo spp. and Nemipterus nematopus escaped through cod-end meshes of $55 \mathrm{~mm}$ and larger, while most Nemipterus bathybus and Upeneus spp. escaped codends of $70 \mathrm{~mm}$ and larger. Nemipterus hexodon, $N$, tambuloides, Lutjanus vittus and Saurida filamentosa mostly escaped the $100-\mathrm{mm}$ cod-end mesh. 

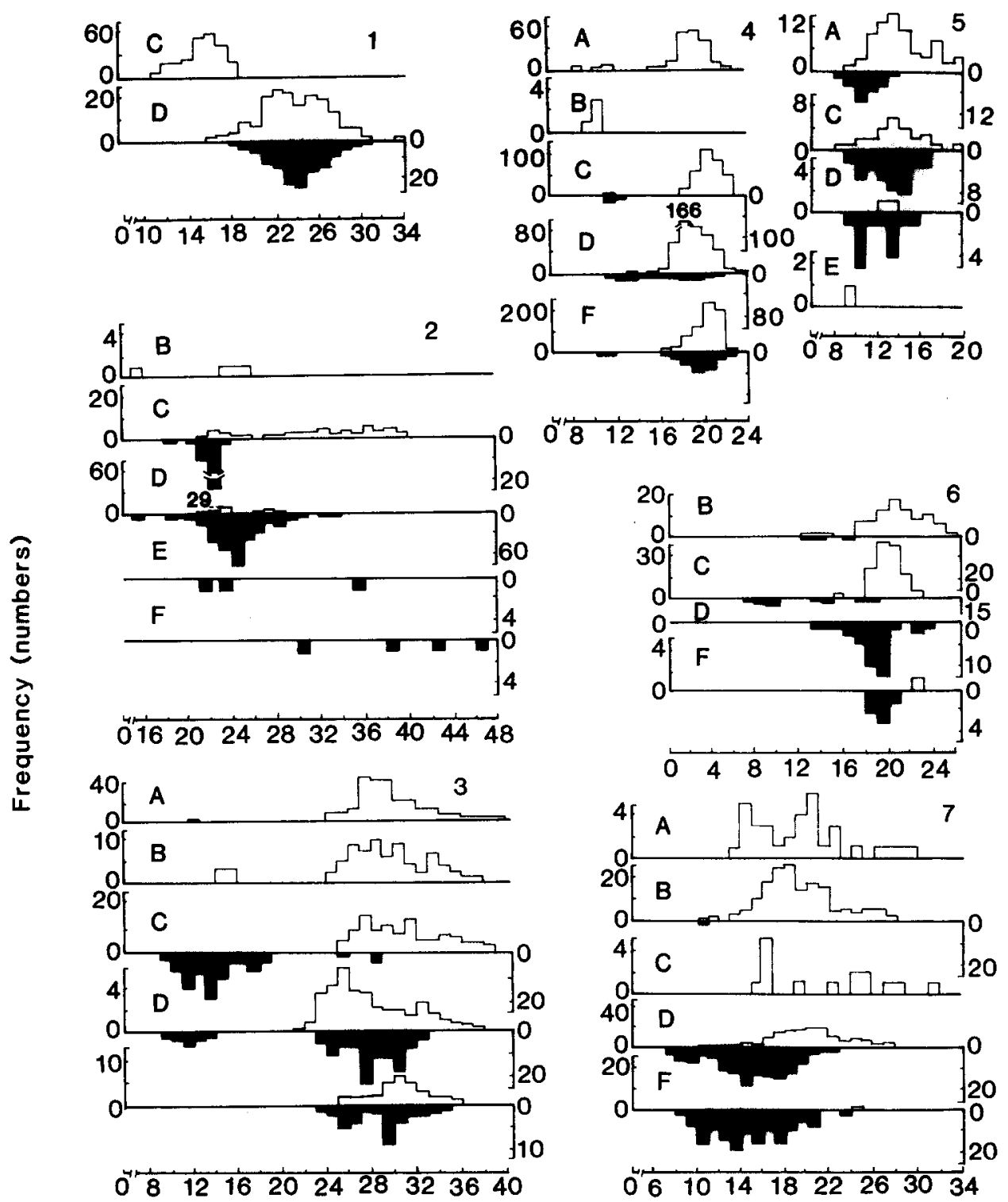

Fig. 5. The length frequency distributions of fishes caught by different codends and cover nets. Codends A-F are defined in Table I (1, Nemipterus tolu; 2, Nemipterus hexadon; 3, Saurida micropectoralis; 4, Sphyraena forsteri; 5 , Leiognathus fasciatus; 6 , Lepidotrigla spiloptera; 7, Trachinocephalus myops). Abscissa, body length; ordinate, number of fishes. Upper histogram for cod-end catches; lower histogram for cover net catches. 


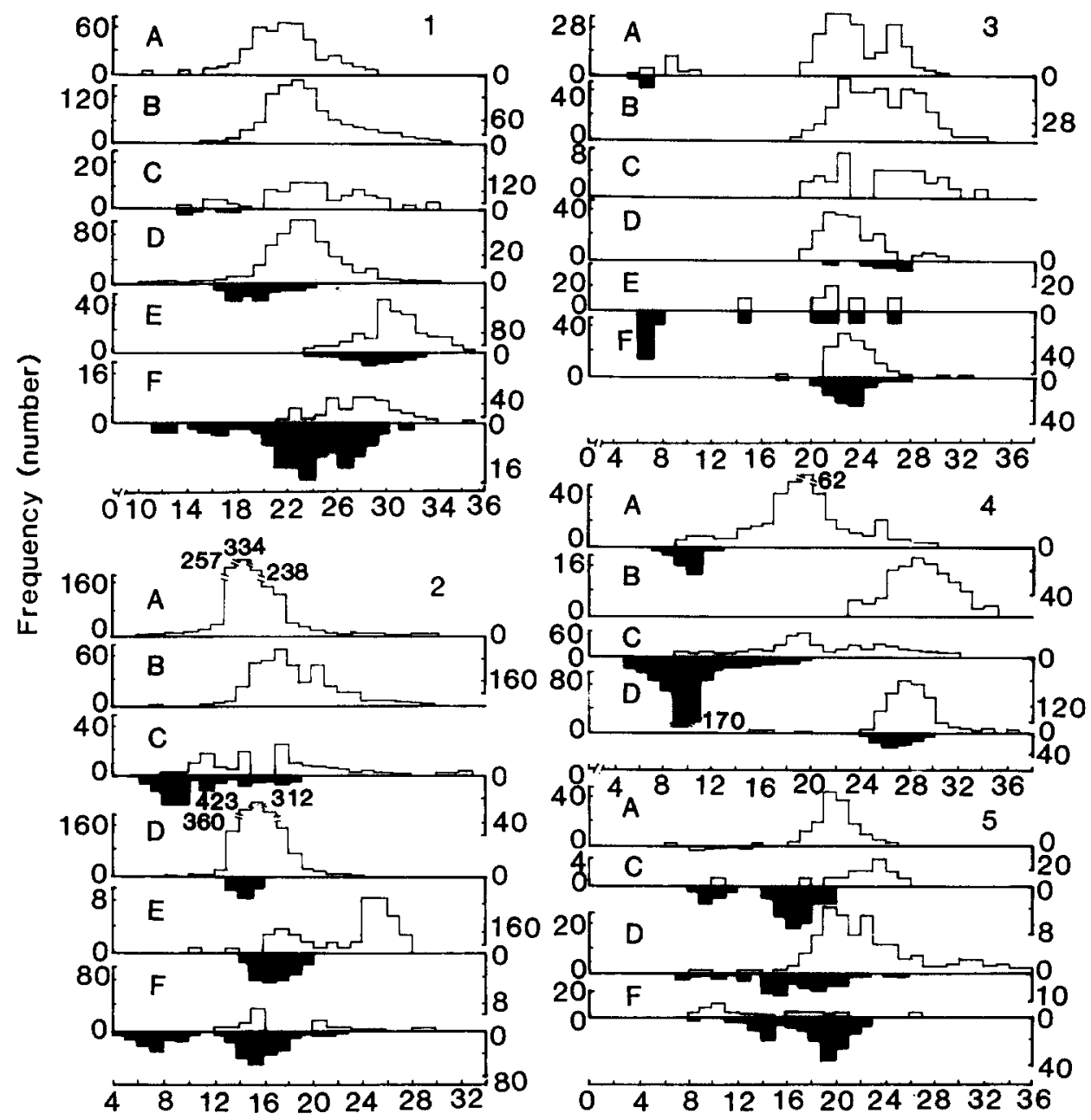

\section{Length $(\mathrm{cm})$}

Fig. 6. The length frequency distributions of fishes caught by different codends and cover nets. Cod-ends A-F are defined in Table I (1, Lutjanus vittus; 2 , Carangoides spp.; 3, Priacanthus hamrur; 4, Johnicops vogleri; 5 , Nemipterus peronii). Abscissa, body length; ordinate, number of fishes. Upper histogram for cod-end catches; lower histogram for cover net catches.

Most of the remaining species showed partial retention by all mesh sizes tested, with both the mean size of retained animals and the proportion escaping increasing with increasing cod-end mesh size (Table III).

Selection factor estimates at three or more mesh sizes were available for 11 species, and scattergrams of the selection factor against mesh size are given for these in Figs. 15 and 16. Selection factors at five or more mesh 

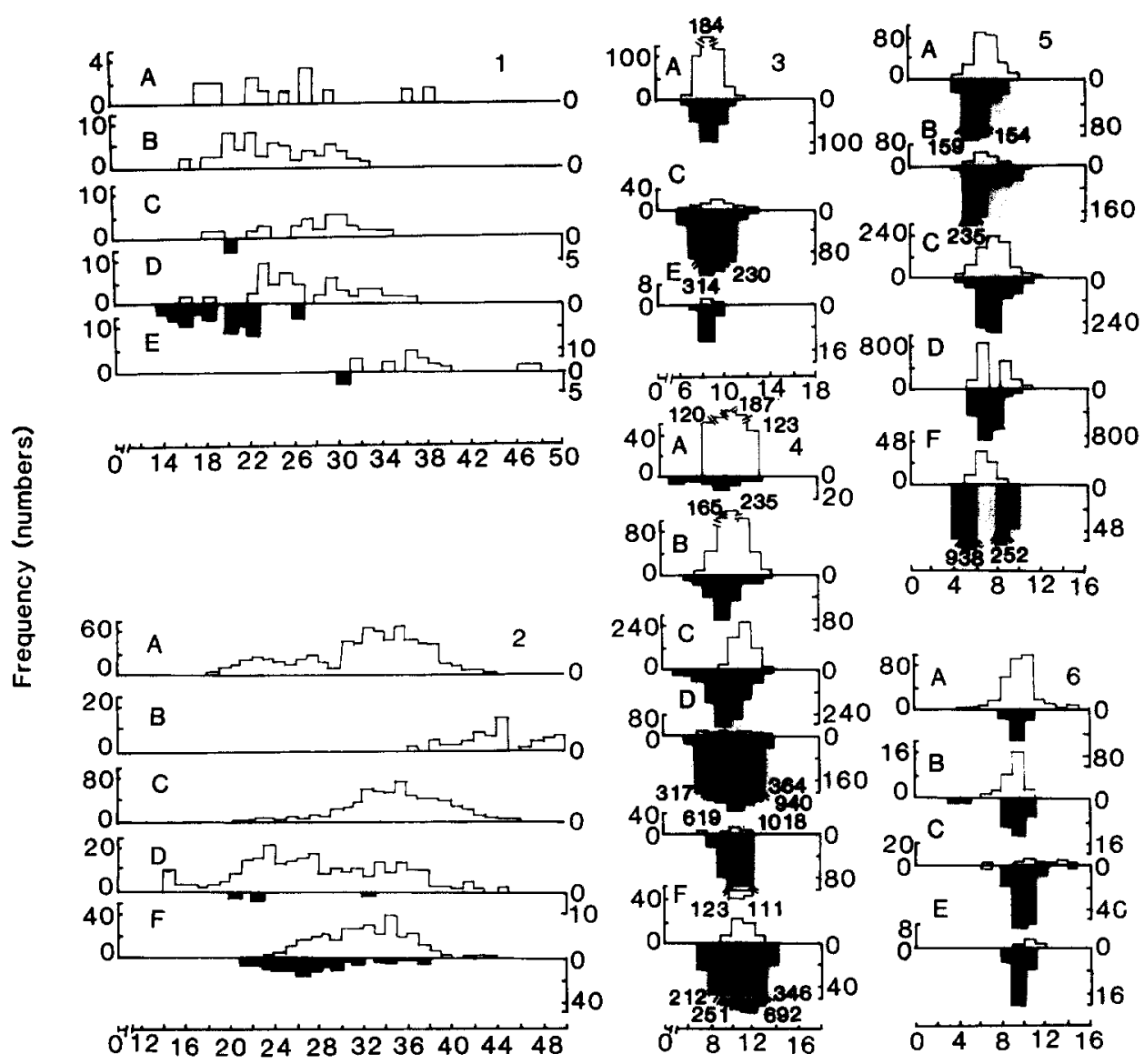

Length $(\mathrm{cm})$

Fig. 7. The length frequency distributions of fishes caught by different cod-ends and cover nets. Cod-ends A-F are defined in Table I (1, Epinephalus spp.; 2, Pomadasys hasta; 3, Acropoma japonicum; 4, Leioganthus spp.; 5, Pentaprion longimanus; 6, Harpodon translucens). Abscissa, body length; ordinate, number of fishes. Upper histogram for cod-end catches; lower histogram for cover net catches.

sizes were available for Nemipterus nematopus, Saurida undosquamis, Parupeneus pleurospilus and Lutjanus vittus, and for these species Spearman's rank correlation coefficient (Snedecor and Cochran, 1967) was also calculated (Fig. 15).

The hypothesis of constant selection factor could be statistically rejected only for S. undosquamis, which showed a significant reduction in selection factor with increasing mesh size. For $S$. undosquamis, the mean size at capture by large-mesh cod-ends is lower than that expected on the basis of selection factors calculated using small-mesh cod-ends (i.e. for a given 


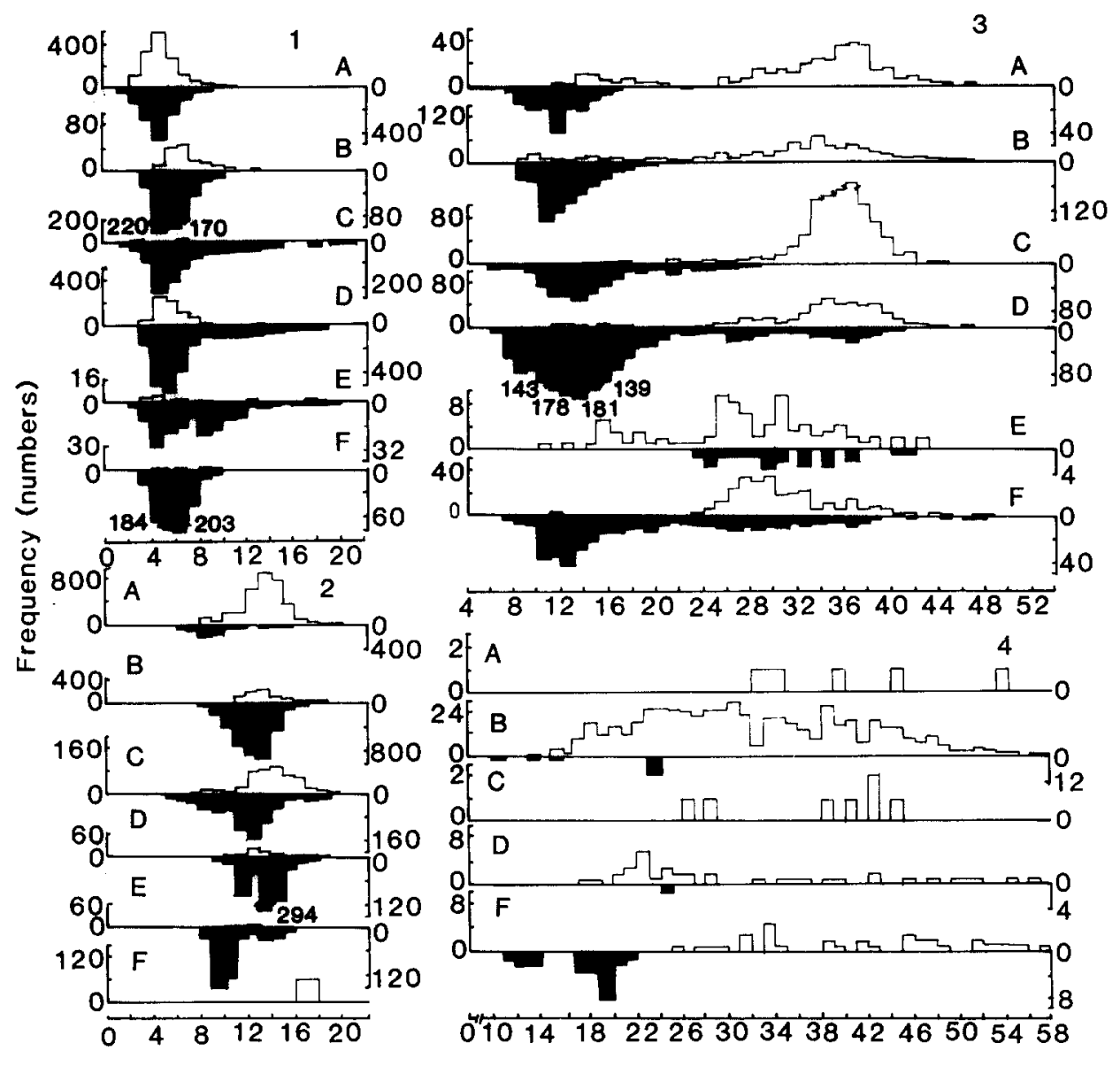

Length $(\mathrm{cm})$

Fig. 8. The length frequency distributions of fishes caught by different cod-ends and cover nets. Cod-ends A-F are defined in Table I (1, Loligo spp.; 2, Upeneus spp.; 3, Saurida undosquamis; 4, Pristipomoides spp.). Abscissa, body length; ordinate, number of fishes. Upper histogram for codend catches; lower histogram for cover net catches.

fish size distribution, fewer fish would escape large-mesh cod-ends than expected by extrapolation of selection factor from tests conducted using small-mesh cod-ends). For $N$. nematopus, $L$. vittus and $P$. pleurospilus there is little indication of a change in selection factor with cod-end mesh size, and the null hypothesis of independence cannot be rejected. Too few data are available to permit statistical analysis for the remainder of the species shown in Figs. 15 and 16, although Nemipterus tambuloides, Upeneus spp. and Leiognathus spp. show some indication of decrease in selection factor with increasing codend mesh size. There is no indication of change in selection factor for Carangoides spp., Nemipterus virgatus and Saurida 


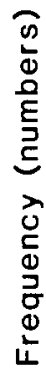
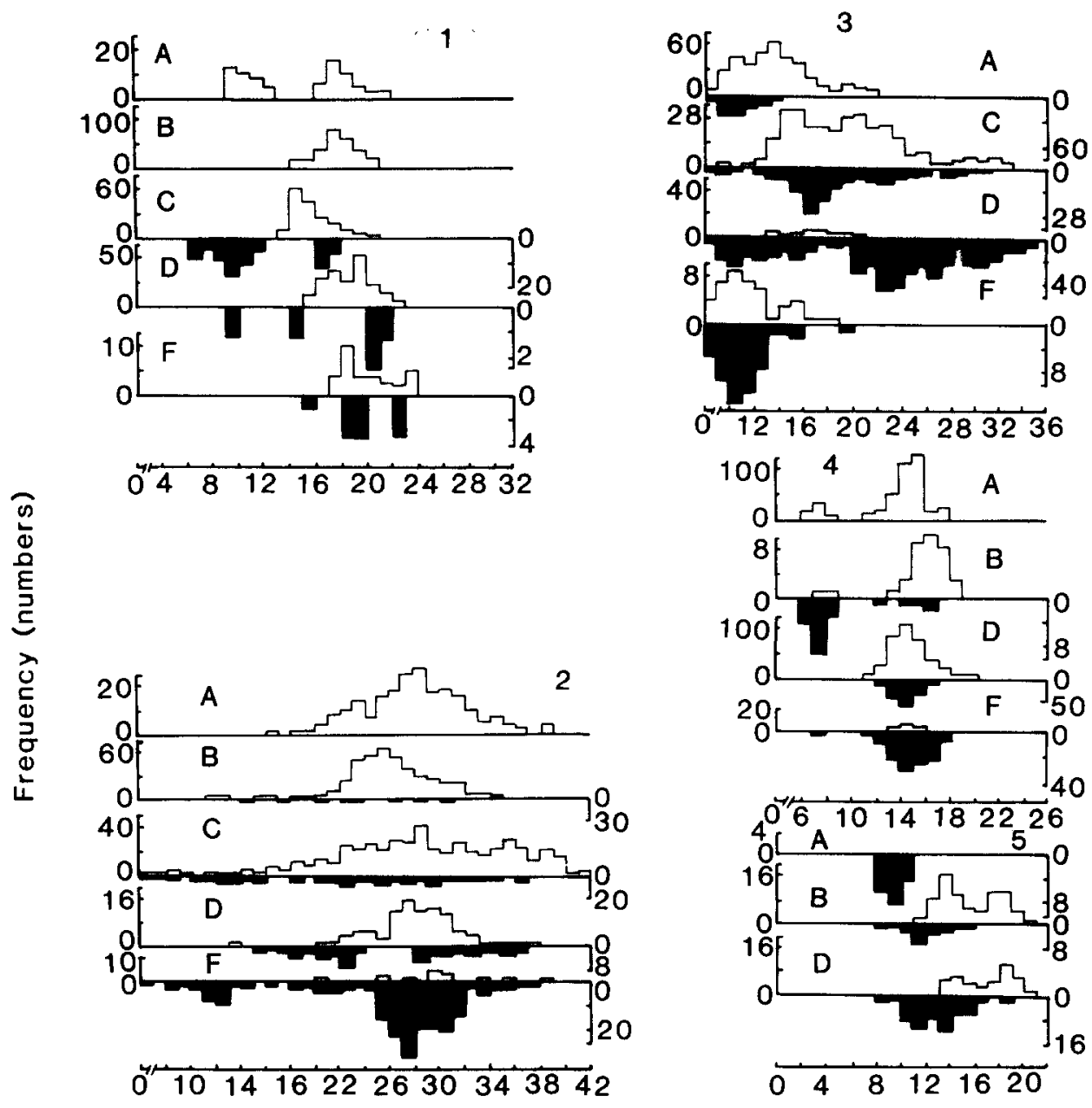

Length $(\mathrm{cm})$

Fig. 9. The length frequency distributions of fishes caught by different cod-ends and cover nets. Cod-ends A-F are defined in Table I (1, Psenopsis anomala; 2 , Saurida filomentosa; 3, Nemipterus bathybus; 4, Therapon theraps; 5, Choerodon monostigma). Abscissa, body length; ordinate, number of fishes. Upper histogram for codend catches; lower histogram for cover net catches.

micropectoralis, and the selection factor of Pentaprion longimanus may increase with cod-end mesh size.

Plots of $L_{0.5}$ against mesh size are given in Fig. 17 for Nemipterus nemotapus, Saurida undosquamis, Lutjanis vittus and Parupeneus pleurospilus. The relationships appear linear, and the parameters of the least squares linear regressions (Snedecor and Cochran, 1967) are given in Table IV. The gradient for all species are significantly greater than zero, and except 
Lutjanus argentimaculatus

Plectorhynchus pictus

Platax batavinus

Formio niger

Myripristus murdjan

Lutjanus sebae

Lutjanus malabaricus

Lutjanus russelli

Priacanthus tayenus

Pomadasys hasta

Lethrinus spp.

Arius thalassinus

Gnathonodon speciosus

siganus canaliculatus

sphyraena forsteri

Adioryx ruber

Argyrops spinifer

saurida micropectoralis

Epinephalus spp.

Lepidotrigla spiloptera

Carangoides spp.

Decapterus maruadsi

Psenopsis anomala

Hapalogenys kishinouyei

cyanoglossus spp.

Seriolina nigrofasciatus

Pristiptis jerdoni

Decapterus maruadsi

Acropoma japonicum

Harpodon translucens

Loligo spp.

Nemipterus nematopus

Nemipterus bathybus

Pentaprion longimanus

Upeneus spp.

Caesio chrysozona

Apogon fasciatus

Nemipterus hexodon

Nemipterus tambuloides

Saurida filomentosa

Leiognathus spp.

Argyrops spinifer

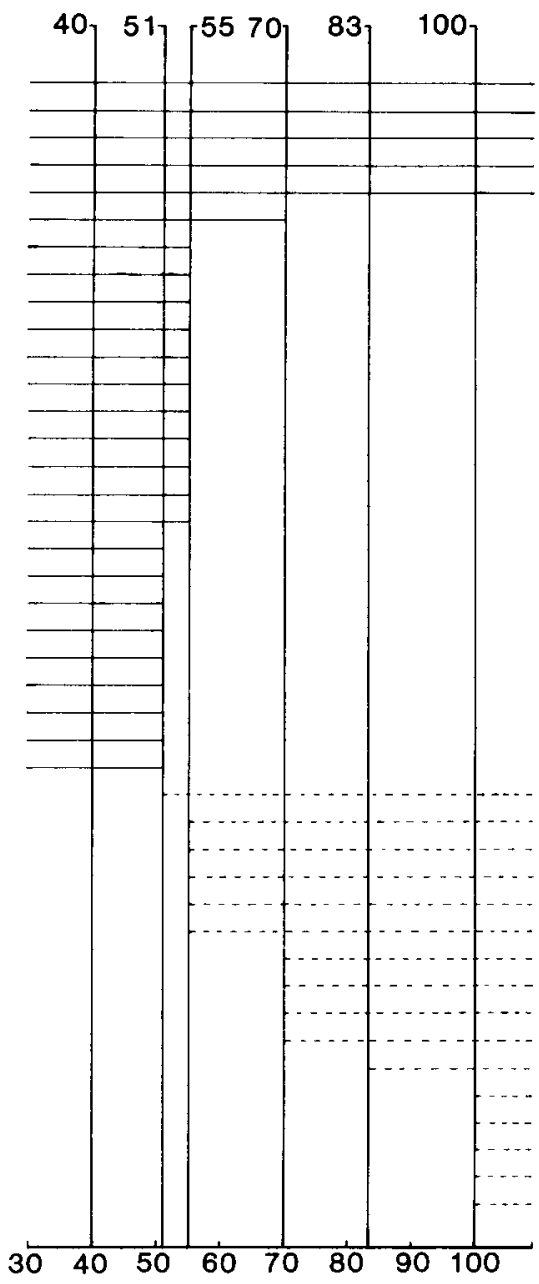

Mesh size (mm)

Fig. 10. The relationship between mesh size and selection rate. Solid lines show the range of mesh size for which the selection rate is zero (total retention in the cod-end), and broken lines show that for which the selection rate is greater than $90 \%$ (less than $10 \%$ retention in the cod-end).

for $S$. undosquamis, the intercepts are not significantly different from zero. For $N$. nematopus, $L$. vittus and $P$. pleurospilus regression lines were recalculated with the constraint that they pass through the origin, and the gradients of these lines provide the best overall estimate of the selection factor for these species.

The detection of significant dependence of selection factor on mesh size for $S$. undosquamis is corroborated by the non-zero intercept of $L_{0.5}$ and mesh size. If the relationship between $L_{0.5}$ and mesh size $(D)$ is given by 


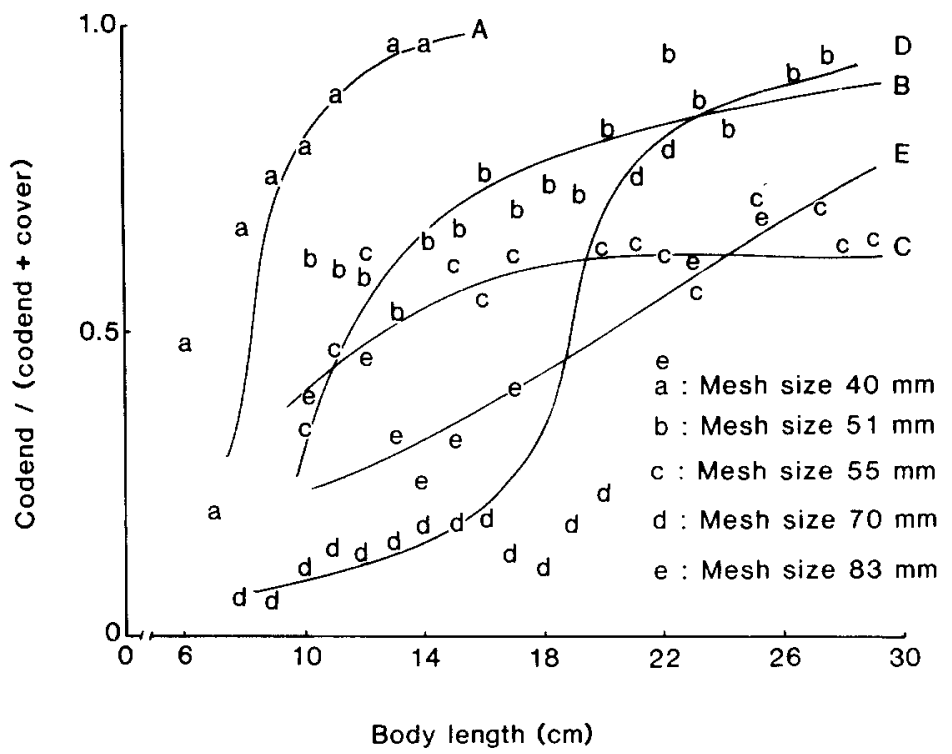

Fig. 11. Fitted logistic selection curves for Nemipterus nematopus for different cod-end mesh sizes. Mesh sizes A-E are defined in Table $I$.

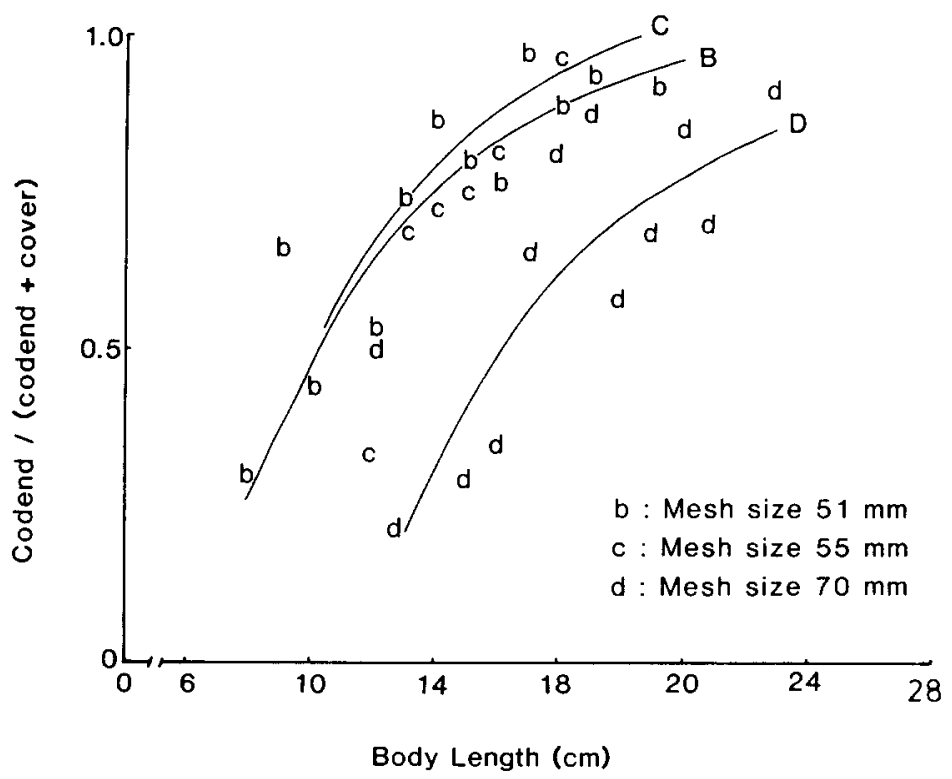

Fig. 12. Fitted logistic selection curves for Nemipterus virgatus for different cod-end mesh sizes. Mesh sizes B-D are defined in Table $I$. 


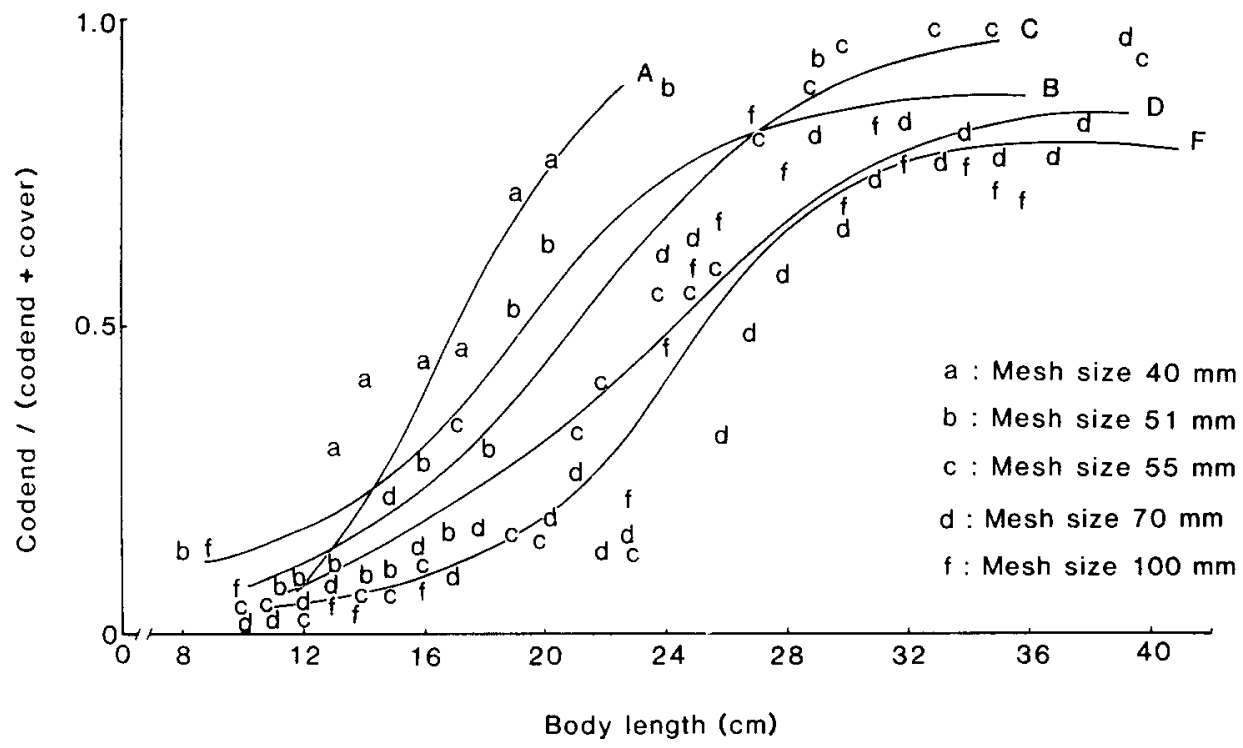

Fig. 13. Fitted logistic selection curves for Saurida undosquamis for different cod-end mesh sizes. Mesh sizes A-F are defined in Table $I$.

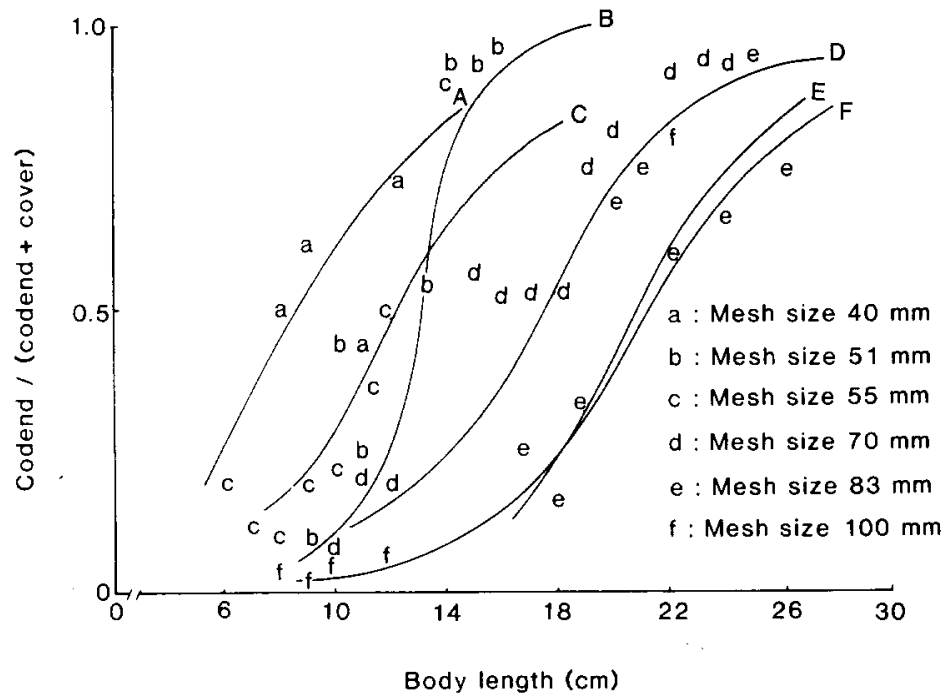

Fig. 14. Fitted logistic selection curves for Parupeneus pleurospilus for different cod-end mesh sizes. Mesh sizes $\mathrm{A}-\mathrm{F}$ are defined in Table $\mathrm{I}$.

$L_{0.5}=a+b D$, then the selection factor $(S)$ is related to mesh size by $S=$ $b+\frac{a}{D}$. This curve is plotted for $S$. undosquamis in Fig. 15 using the regression parameters in Table IV. Thus change in $S$ for this species is due to a non-zero intercept, rather than to non-linearity in the relationship between 

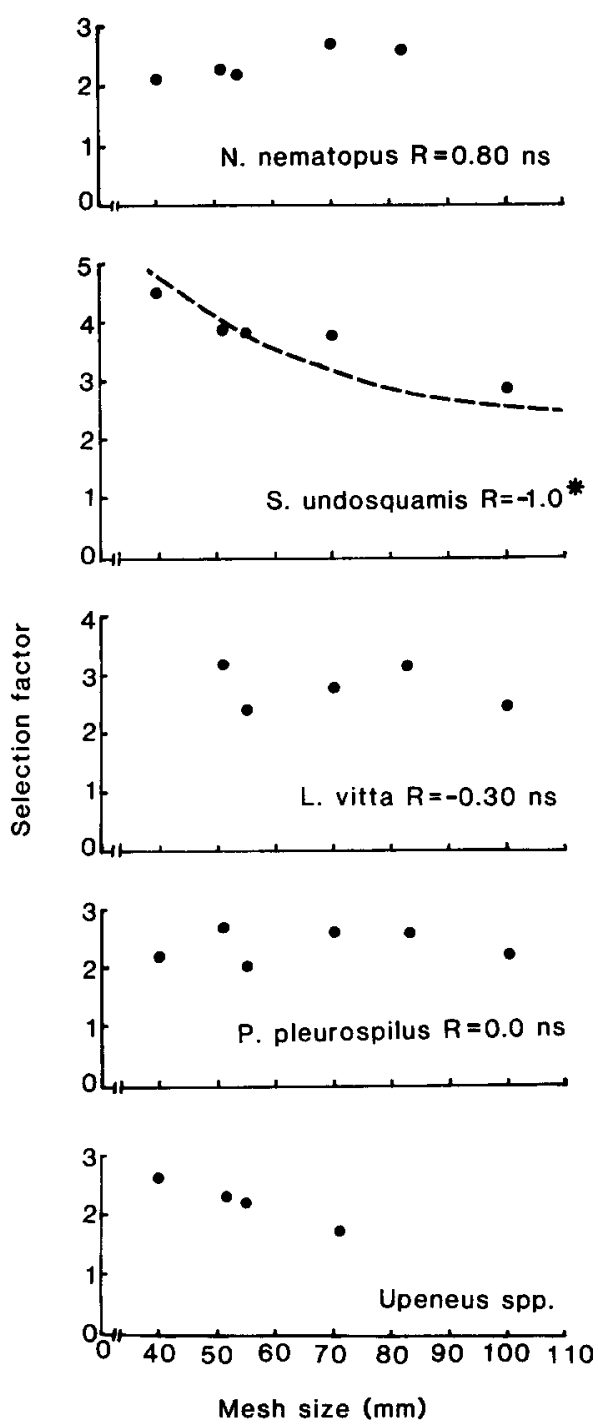

Fig. 15. Scattergrams of selection factor and codend mesh size (based on data in Table III). $R$ is Spearman's rank correlation coefficient. ns indicates not significantly different from zero at $\alpha=0.05 ; *$ indicates significantly different from zero at $\alpha=0.05$. The line shown for Saurida undosquamis was derived from the linear regression analysis of $L_{0.5}$ against mesh size (see text).

$L_{0.5}$ and mesh size within the range of mesh sizes examined (although presumably some non-linearity exists for mesh sizes smaller than $40 \mathrm{~mm}$ ).

\section{DISCUSSION}

Selection factors given by Jones (1976) and Sinoda et al. (1979) for the same or similar species from the South China Sea are listed in Table V. 

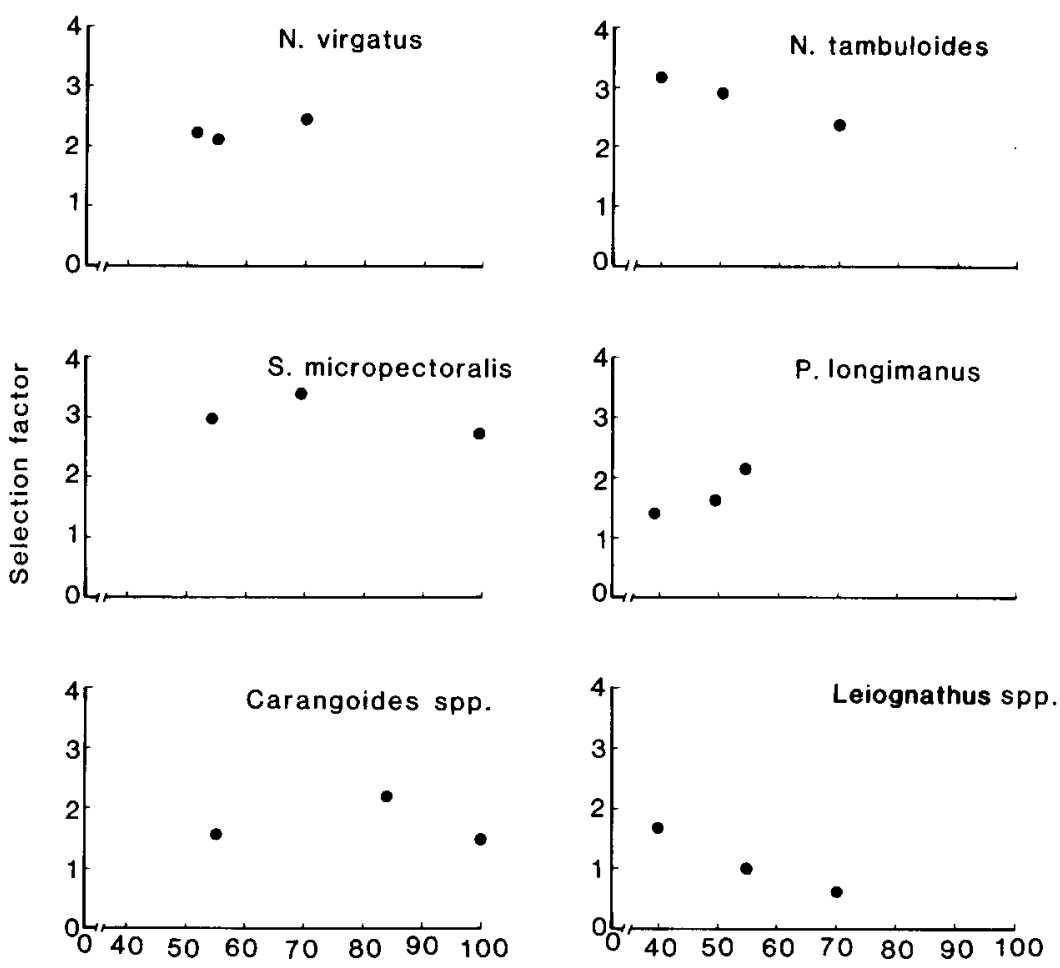

Mesh size $(\mathrm{mm})$

Fig. 16. Scattergrams of selection factor and cod-end mesh size (based on data on Table III).

\section{TABLE IV}

Least squares linear regression for the relationship between $L_{0,5}$ and cod-end mesh size, and Student $t$ values for test of the hypotheses that the gradient is zero $\left(t_{b}\right)$ and that the intercept is zero $\left(t_{a}\right)$. For Nemipterus nematopus, Lutjanus vittus and Parupeneus pleurospilus the estimate of the gradient of regression through the origin is also given. ns $=$ not significant at $\alpha=0.05 ;^{*}=$ significant at $\alpha=0.05 ; * *=$ significant at $\alpha=0.01$.

\begin{tabular}{lrllll}
\hline & $\begin{array}{l}\text { Intercept } \\
(\mathrm{mm})\end{array}$ & \multicolumn{1}{c}{$t_{\mathfrak{a}}$} & Gradient & \multicolumn{1}{c}{$t_{b}$} & $\begin{array}{l}\text { Gradient } \\
\text { (through origin) }\end{array}$ \\
\hline N. nematopus & -42.74 & $2.58 \mathrm{~ns}$ & 3.11 & $11.86^{* *}$ & 2.45 \\
L. vittus & 19.88 & $0.41 \mathrm{~ns}$ & 2.50 & $3.80^{*}$ & 2.75 \\
P. pleurospilus & 7.73 & $0.30 \mathrm{~ns}$ & 2.27 & $6.19^{*}$ & 2.38 \\
S. undosquamis & 134.78 & $5.10^{*}$ & 1.36 & $3.41^{*}$ & \\
\hline
\end{tabular}



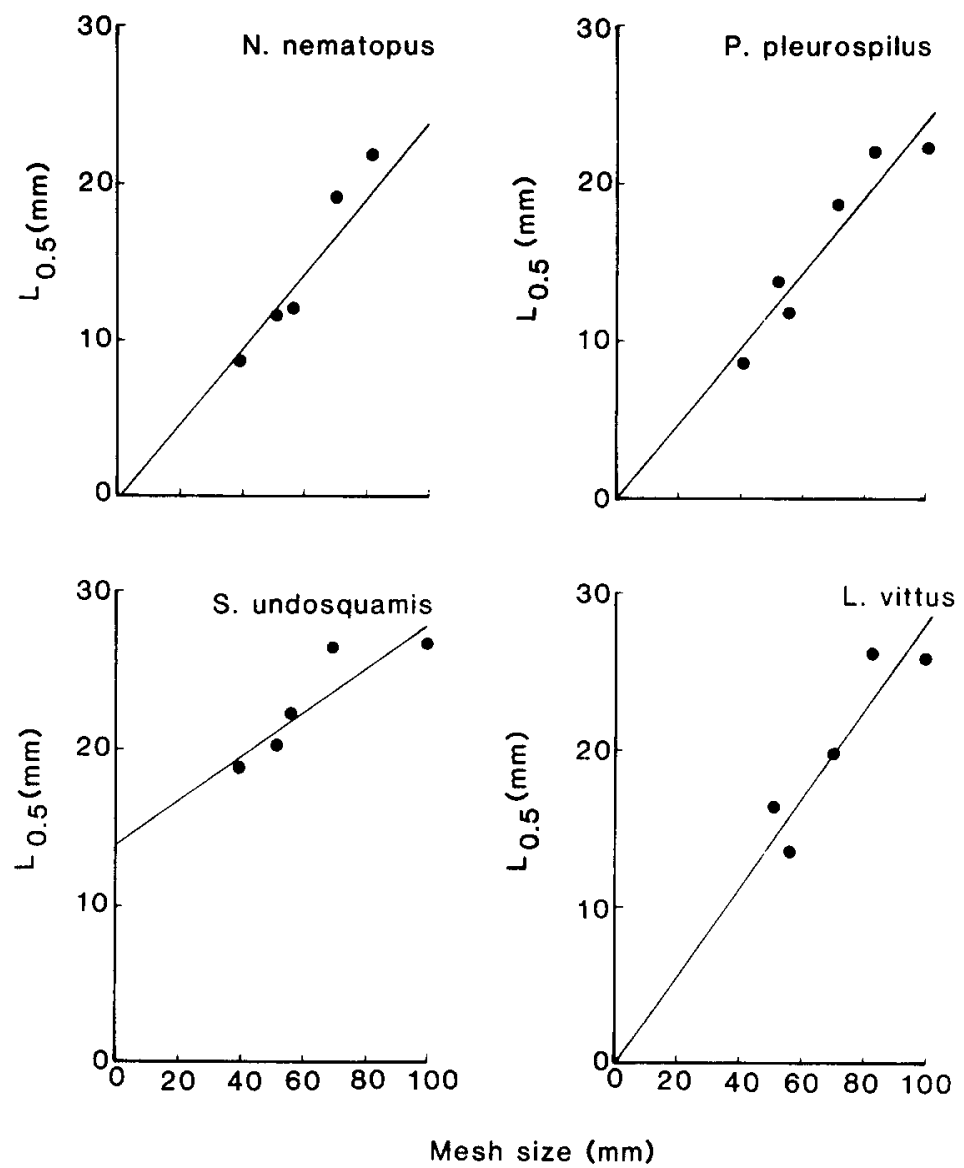

Fig. 17. Fish length at $50 \%$ retention $\left(L_{0,5}\right)$ plotted against cod-end mesh size. The least squares regression lines show for Nemipterus nematopus, Parupeneus pleurospilus and Lutjanus vittus are constrained to pass through the origin, while that for Saurida undosquamis is not. The regression line parameters are given in Table IV.

There is generally good agreement between the selection factor estimates obtained from the South China Sea and those provided here from northern Australia. The greatest discrepancies are among the Nemipterus species (selection factor $2.4-3.8$ in the literature and 1.9-2.5 in this study), Leiognatus species (selection factor 1.9-3.0 in Sinoda et al. (1979) and 0.7-1.7 in this study) and Upeneus spp. (2.7-2.9 in the literature and 1.8-2.6 here). It is not known why these species were retained at a smaller size in our experiment than in previous selectivity experiments.

Nemipterus spp. and Saurida spp. are the two largest components of catch from the North West Shelf of Australia (Liu et al., 1978), and the present minimum mesh size of $60 \mathrm{~mm}$ implies a length at $50 \%$ retention of about 14 and $22 \mathrm{~cm}$ for these species, respectively. Squid (mostly Loligo spp.) also constitute an important component of the commercial catch, 


\section{TABLE V}

Values of the selection factor reported for species which are the same or similar to those studied here

\begin{tabular}{lll}
\hline & Jones (1976) & Sinoda et al. (1979) \\
\hline Saurida undosquamis & 3.7 & 3.6 \\
S. micropectoralis & & 4.4 \\
Nemipterus hexodon & 3.8 & \\
N. japonicus & 2.4 & 3.2 \\
N. marginatus & 2.9 & 2.7 \\
N. peronii & & 3.0 \\
Priacanthus tayenus & & 1.9 \\
P. macracanthus & & 2.3 \\
Lutjanus vittus & & 2.3 \\
Carangoides malabaricus & & 2.0 \\
Parupeneus pleurospilus & & 2.5 \\
Upeneus sulphureus & 2.7 & 2.7 \\
U. bensasi & & 2.9 \\
Leiognathus bindus & & 2.0 \\
Sphyraena jello & 3.7 & \\
\hline
\end{tabular}

particularly in the Timor and Arafura Seas (Liu et al., 1978), and the selection experiments show that significant quantities of squid would escape through the currently used cod-ends. Escape through a $60-\mathrm{mm}$ cod-end would be negligible for most of the Lutjanus, Lethrinus, Pomadasys and Pristipomoides species, and also for Argyrops spinifer, Priacanthus tayenus and Plectorhynchus pictus. It is clear that the use of a single mesh size for the fishery as a whole is a compromise in which some species will have very little chance of escape through the cod-end, while others will have little chance of being retained. This problem is examined further by Sainsbury (1984).

The effects of different cod-end mesh size on $L_{0.5}$ and selection factor could be examined in detail only for Nemipterus nematopus, Saurida undosquamis, Lutjanus vittus and Parupeneus pleurospilus. The assumption of direct proportionality of $L_{0.5}$ with mesh size, and the resulting independence of selection factor and mesh size, was rejected for only one species, $S$. undosquamis. While this finding indicates that the assumption is probably acceptable for many species, the exception provided by $S$. undosquamis illustrates that important species-specific variation exists. Change in the selection factor-mesh size relationship of $S$. undosquamis is greatest at small mesh sizes (less than about $70 \mathrm{~mm}$ ), which is the region of greatest relevance to most existing trawl fisheries in the Indo-Pacific region (Jones, 1976). Non-linearity of selection factor at small mesh size is expected whenever the relationship between $L_{0.5}$ and mesh size has a non-zero intercept, even if that relationship is linear over the range of mesh sizes examined. 
There are indications that selection factor may also increase with mesh size for Upeneus spp., Leioganthus spp. and Nemipterus tambuloides. The phenomenon could be widespread and warrants further investigation.

Relationship between $L_{0.5}$ and mesh size other than direct proportionality are easily incorporated into fisheries models used to examine the effects of changed mesh size (e.g. Beverton and Holt, 1957; Sainsbury, 1984). However, in situations other than direct proportionality the selection factor becomes unnecessarily complex, and in general analysis should proceed directly from the established relationship between $L_{0.5}$ and mesh size.

\section{REFERENCES}

Anonymous, 1979. Annual report of effort and catch statistics by area on Taiwan demersal fish fisheries 1978. Demersal Fish Research Centre, Taipei, Taiwan.

Anonymous, 1980. Annual report of effort and catch statistics by area on Taiwan demersal fish fisheries 1979. Demersal Fish Research Centre, Taipei, Taiwan.

Aoyoma, T., 1961. The selective action of trawl nets and its application to the management of the Japanese trawl fisheries in the East China and the Yellow Seas. Bull. Seikai Reg. Fish. Res. Lab., 23:63 pp.

Beverton, R.J.H. and Holt, S.J., 1957. On the dynamics of exploited fish populations. U.K. Ministry of Agriculture and Fisheries, Fishery Investigation Series II, Vol. XIX.

Bliss, C.I., 1970. Statistics in Biology, Volume 2. McGraw-Hill, New York.

Clark, J.R., 1963. Size selection of fish by otter trawls. Results of recent experiments in the northwest Atlantic. In: The Selectivity of Fishing Gear. ICNAF Spec. Publ. 5, pp. 25-96.

Jensen, A.J.C., 1949. The relations between the size of mesh and the length of fish released. Rapp. P.-V. Reun. Cons. Int. Explor. Mer, 125: 65-69.

Jones, R., 1976. Mesh regulation in the demersal fisheries of the South China Sea area. South China Sea Fisheries Development and Coordinating Programme, SCS/76/WP/34, $75 \mathrm{pp}$.

Liu, H.S., Lai, L.H. and Yeh, S.Y., 1978. General review of demersal fish resources in the Sunda Shelf and the Australian Waters. Acta Oceanogr. Taiwanica, 8: 109-140.

Pope, J.A., 1975. Manual of methods for fish stock assessment. Part 3. Selectivity of fishing gear. FAO Fisheries Technical Paper 41 (Revision 1), 46 pp.

Sainsbury, K.J., 1984. Optimal mesh size for tropical multispecies trawl fisheries. J. Cons., Cons. Int. Explor. Mer, 41: 129-139.

Sinoda, M., Tan, S.M., Watanabe, Y. and Meemeskul, Y., 1979. A method for estimating the best codend mesh size in the South China Sea area. Bull. Choshi Mar. Lab., Chiba University, 11: 65-80.

Snedecor, G.W. and Cochran, W.G., 1967. Statistical Methods. 6th edn. Iowa State University Press. 